TRANSACTIONS OF THE

AMERICAN MATHEMATICAL SOCIETY

Volume 361, Number 2, February 2009, Pages 1103-1127

S 0002-9947(08)04599-6

Article electronically published on September 9, 2008

\title{
BETTI NUMBERS FOR FAT POINT IDEALS IN THE PLANE: A GEOMETRIC APPROACH
}

\author{
ALESSANDRO GIMIGLIANO, BRIAN HARBOURNE, AND MONICA IDÀ
}

\begin{abstract}
We consider the open problem of determining the graded Betti numbers for fat point subschemes $Z$ supported at general points of $\mathbf{P}^{2}$. We relate this problem to the open geometric problem of determining the splitting type of the pullback of $\Omega_{\mathbf{P}^{2}}$ to the normalization of certain rational plane curves. We give a conjecture for the graded Betti numbers which would determine them in all degrees but one for every fat point subscheme supported at general points of $\mathbf{P}^{2}$. We also prove our Betti number conjecture in a broad range of cases. An appendix discusses many more cases in which our conjecture has been verified computationally and provides a new and more efficient computational approach for computing graded Betti numbers in certain degrees. It also demonstrates how to derive explicit conjectural values for the Betti numbers and how to compute splitting types.
\end{abstract}

\section{INTRODUCTION}

Let $Z=m_{1} P_{1}+\cdots+m_{n} P_{n}$ be a fat point subscheme of $\mathbf{P}^{2}$ supported at general points $P_{i}$. Thus $Z$ is the 0 -dimensional subscheme of $\mathbf{P}^{2}$ defined by the homogeneous ideal $I(Z)=\bigcap_{i} I\left(P_{i}\right)^{m_{i}}$ in the homogeneous coordinate ring $R=K\left[\mathbf{P}^{2}\right]$ of $\mathbf{P}^{2}$ (where we take $K$ to be an algebraically closed field of arbitrary characteristic), where $I\left(P_{i}\right)$ is the ideal generated by all homogeneous forms $f \in R$ vanishing at $P_{i}$. The homogeneous component $I(Z)_{t}$ of $I(Z)$ in degree $t$ is just the $K$-vector space span of the homogeneous elements of $I(Z)$ of degree $t$. Thus $I(Z)_{t}$ consists of all homogeneous polynomials of degree $t$ which vanish at each point $P_{i}$ to order at least $m_{i}$. The Hilbert function of $I(Z)$ is the function $h_{Z}$ which gives the $K$-vector space dimension $h_{Z}(t)=\operatorname{dim} I(Z)_{t}$ of $I(Z)_{t}$ as a function of $t$.

1.1. The SHGH conjecture. The problem of determining $h_{Z}$ has attracted a lot of attention over the years, and it is still an open problem in general. In fact, even the most fundamental problem is open: it is not known in general what the least $t$ is (which we call $\alpha(Z)$ ) for which $I(Z)_{t} \neq 0$. The fact that even the latter problem is open is less surprising given that if one knows $\alpha(Z)$ for every $Z$, then one can determine $h_{Z}(t)$ for all $t$ and $Z$, and conversely.

Given that it is not known how big $I(Z)_{t}$ is, or even if it is nonzero, it is not surprising that the least value of $t$ such that $I(Z)$ is generated in degrees $t$ or less is not known. More precisely, the graded Betti numbers for the minimal free resolution of $I(Z)$ are not known. There is not even a conjecture in general for the

Received by the editors December 29, 2006 and, in revised form, June 15, 2007.

2000 Mathematics Subject Classification. Primary 14C20, 13P10; Secondary 14J26, 14J60.

Key words and phrases. Graded Betti numbers, fat points, splitting types.

(C)2008 American Mathematical Society

Reverts to public domain 28 years from publication 
Betti numbers. On the other hand, there is a general conjecture for the values of the Hilbert function $h_{Z}$. This is the SHGH Conjecture (due, in various equivalent forms, to Segre [S], Harbourne [Ha4], Gimigliano G] and Hirschowitz [Hi1; see Conjecture 2.2.1 or Conjecture A1.2.1). Considerations of geometry lead to a lower bound $e\left(h_{Z}, t\right)$ for $h_{Z}(t)$ (the definition of $e\left(h_{Z}, t\right)$, which is somewhat complicated, is given in the appendix). The SHGH Conjecture asserts that $e\left(h_{Z}, t\right)=h_{Z}(t)$. Thus $e\left(h_{Z}, t\right)$ is regarded as the "expected" value of $h_{Z}(t)$.

In degrees $t>\alpha(Z)$, the SHGH Conjecture implies the following simple statement:

Conjecture 1.1.1. Let $Z=m_{1} P_{1}+\cdots+m_{n} P_{n}$ for nonnegative integers $m_{i}$ and general points $P_{i} \in \mathbf{P}^{2}$. If $t>\alpha(Z)$ and $f_{1}^{b_{1}} \cdots f_{r}^{b_{r}}$ is a factorization of the greatest common divisor of $I(Z)_{t}$ as a product of nonassociate irreducible factors, then

$$
h_{Z}(t)=\left(\begin{array}{c}
t+2 \\
2
\end{array}\right)-\sum_{i}\left(\begin{array}{c}
m_{i}+1 \\
2
\end{array}\right)+\sum_{l}\left(\begin{array}{c}
b_{l} \\
2
\end{array}\right) .
$$

In particular, under the assumptions given, $e\left(h_{Z}, t\right)=\left(\begin{array}{c}t+2 \\ 2\end{array}\right)-\sum_{i}\left(\begin{array}{c}m_{i}+1 \\ 2\end{array}\right)+$ $\sum_{l}\left(\begin{array}{c}b_{l} \\ 2\end{array}\right)$. Although it is perhaps not clear from this how to actually compute $e\left(h_{Z}, t\right)$, the full SHGH Conjecture allows one to compute $e\left(h_{Z}, t\right)$ in terms only of the $m_{i}$, and also to conjecturally determine the degrees and multiplicities at each point $P_{i}$ of all curves in the base locus of $I(Z)_{t}$. The advantage of the statement above is that it gives insight into the problem of determining $h_{Z}$, without the burden of the technicalities needed to state the full SHGH Conjecture.

For example, $I(Z)_{t}$ consists of all homogeneous polynomials of degree $t$ which vanish at each point $P_{i}$ to order at least $m_{i}$. But the vector space of all forms of degree $t$ has dimension $\left(\begin{array}{c}t+2 \\ 2\end{array}\right)$, and requiring vanishing at $P_{i}$ to order $m_{i}$ imposes $\left(\begin{array}{c}m_{i}+1 \\ 2\end{array}\right)$ independent linear conditions. We do not know that the conditions imposed at one point are independent of those imposed at all of the other points, and in fact they are not always independent. Thus we obtain the bound $h_{Z}(t) \geq$ $\left(\begin{array}{c}t+2 \\ 2\end{array}\right)-\sum_{i}\left(c_{i}^{m_{i}+1}\right)$. What the SHGH Conjecture does in essence is to give a precise measure of the failure of the imposed conditions to be independent. The key insight is that the failure of independence in degree $t>\alpha(Z)$ is due to the gcd not being square-free. What the conjecture above hides is that the curves defined by the forms $f_{i}$ are thought always to be very special, and this is significant for what we do in this paper.

To make this clearer we strengthen the conjecture above to include the case that $t=\alpha(Z)$. To do this we need a minor technicality. We say a plane curve $C$ defined by an irreducible form of degree $d$ is contributory with respect to points $P_{1}, \ldots, P_{n}$ if $C$ is a rational curve smooth except possibly at the points $P_{i}$, such that mult $_{P_{1}}(C)+\cdots+$ mult $_{P_{n}}(C)=3 d-1$. We will say a plane curve $C$ defined by an irreducible form of degree $d$ is negative with respect to points $P_{1}, \ldots, P_{n}$ if $\left(\text { mult }_{P_{1}}(C)\right)^{2}+\cdots+\left(\text { mult }_{P_{n}}(C)\right)^{2}>d^{2}$. It follows by the genus formula that curves contributory for $P_{1}, \ldots, P_{n}$ are also negative.

After extending Conjecture 1.1.1, we have:

Conjecture 1.1.2. Let $Z=m_{1} P_{1}+\cdots+m_{n} P_{n}$ for nonnegative integers $m_{i}$ and general points $P_{i} \in \mathbf{P}^{2}$. Given $t \geq \alpha(Z)$, let $f_{1}^{c_{1}} \cdots f_{r}^{c_{r}}$ be a factorization of the greatest common divisor of $I(Z)_{t}$ as a product of nonassociate irreducible factors. Then every factor $f_{j}$ which defines a curve negative for the points $P_{i}$ defines a 
contributory curve, and we have

$$
h_{Z}(t)=\left(\begin{array}{c}
t+2 \\
2
\end{array}\right)-\sum_{i}\left(\begin{array}{c}
m_{i}+1 \\
2
\end{array}\right)+\sum_{k}\left(\begin{array}{c}
c_{j_{k}} \\
2
\end{array}\right),
$$

where the index $k$ runs over the factors $f_{j_{k}}$ defining curves contributory for the points $P_{i}$.

The point is that the SHGH Conjecture implies that it is only exponents $c_{j}$ of factors $f_{j}$ defining contributory curves which contribute to the lack of independence. The SHGH Conjecture also implies in degrees $t>\alpha(Z)$ that every factor $f_{j}$ defines a contributory curve, and that every curve negative for general points is in fact contributory. (Factors defining noncontributory curves do occur in degree $\alpha$, however; for example, if $Z=P_{1}+\cdots+P_{9}$ consists of 9 general points, then $\alpha(Z)=3$ and the gcd in degree 3 is an irreducible cubic defining an elliptic curve $C$, which is therefore not contributory, but it is also not negative.)

It is known, however, that contributory curves do contribute to the failure of independence. The SHGH Conjecture is that nothing else contributes. In particular, if the greatest common divisor of $I(Z)_{t}$ is 1 (i.e., if the zero locus of $I(Z)_{t}$ is at most 0-dimensional), or more generally if the gcd of $I(Z)_{t}$ is just square-free, then the conjecture is that there is no failure of independence, and hence that $h_{Z}(t)=\left(\begin{array}{c}t+2 \\ 2\end{array}\right)-\sum_{i}\left(\begin{array}{c}m_{i}+1 \\ 2\end{array}\right)$.

1.2. The Betti number conjecture. The goal of this paper is to give a theorem and a conjecture for graded Betti numbers for ideals $I(Z)$, mimicking the fact that there is a bound $e\left(h_{Z}, t\right) \leq h_{Z}(t)$ which by the SHGH Conjecture is an equality, given explicitly by Conjecture 1.1.2.

The SHGH Conjecture specifies how big the ideal $I(Z)$ is in each degree. The next question, for which no general conjecture has yet been posed, is where must one look for generators of $I(Z)$. More precisely, in any minimal set of homogeneous generators, how many generators are there in each degree? The goal of this paper is to develop (and prove cases of) a conjecture for the numbers of generators in each degree greater than $\alpha(Z)+1$. The number of generators in degrees less than $\alpha(Z)+1$ is trivial: there are obviously no generators in degrees $t<\alpha(Z)$, and, since any minimal set of homogeneous generators of $I(Z)$ must include a $K$-vector space basis of $I(Z)_{\alpha}$, there are precisely $h_{Z}(t)$ generators in degree $t=\alpha(Z)$. There remains the question of how many generators there are in degree $t=\alpha(Z)+1$. Our approach is to relate the number of generators in a given degree to the splitting of a certain rank 2 bundle on certain curves. In degree $\alpha(Z)+1$ precisely what curves must be taken into account is more subtle than it is in larger degrees. Thus here we focus on degrees larger than $\alpha(Z)+1$. We study the subtleties needed for a unified approach that subsumes degree $\alpha(Z)+1$ in separate papers, beginning with GHI1.

For any $t$, the number of homogeneous generators in degree $t+1$ in any minimal set of homogeneous generators is the dimension of the cokernel of the map $\mu_{t}$ : $I(Z)_{t} \otimes R_{1} \rightarrow I(Z)_{t+1}$ given on simple tensors by multiplication, $f \otimes g \mapsto f g$. What we will do is to give a conjecture for $\operatorname{dim}$ cok $\mu_{t}$ for all $Z$ in all degrees $t>\alpha(Z)$. Clearly $\operatorname{dim} \operatorname{cok} \mu_{t}=0$ if $t<\alpha(Z)-1$ and $\operatorname{dim} \operatorname{cok} \mu_{t}=h_{Z}(\alpha(Z))$ if $t=\alpha(Z)-1$, so our conjecture handles all cases except $t=\alpha(Z)$. The conjecture is in terms of data determined by contributory curves. 
Let $C^{\prime}$ be a curve contributory for points $P_{1}, \ldots, P_{n}$. The composition of the normalization map $C \rightarrow C^{\prime}$ with the inclusion $C^{\prime} \subset \mathbf{P}^{2}$ gives a morphism $f$ : $C \rightarrow \mathbf{P}^{2}$. Pulling back the twisted cotangent bundle $\Omega_{\mathbf{P}^{2}}(1)$ gives a rank two bundle $f^{*}\left(\Omega_{\mathbf{P}^{2}}(1)\right)$ on $C$, but $C$ is smooth and rational, so $f^{*}\left(\Omega_{\mathbf{P}^{2}}(1)\right)$ splits as $f^{*}\left(\Omega_{\mathbf{P}^{2}}(1)\right) \cong \mathcal{O}_{C}\left(-a_{C}\right) \oplus \mathcal{O}_{C}\left(-b_{C}\right)$ for some integers $a_{C} \leq b_{C}$. We call $\left(a_{C}, b_{C}\right)$ the splitting type of $C$ (and for convenience, we set $a_{C^{\prime}}=a_{C}$ and $b_{C^{\prime}}=b_{C}$ and refer to $\left(a_{C^{\prime}}, b_{C^{\prime}}\right)$ also as the splitting type of $\left.C^{\prime}\right)$.

Given $t>\alpha(Z)$, clearly $I(Z)_{t-1} \neq 0$, so $I(Z)_{t-1}$ has a well-defined gcd (up to a scalar multiple). Let $\gamma_{t-1}$ be the product of all irreducible factors of the gcd defining curves negative for the points $P_{1}, \ldots, P_{n}$. (If $t>\alpha(Z)+1$, then, as in Conjecture 1.1.1, the SHGH Conjecture implies that $\gamma_{t-1}$ is itself the gcd.) Let $\gamma_{t-1}=f_{1}^{c_{1}} \cdots f_{r}^{c_{r}}$ be its factorization into nonassociate irreducible factors and let $d_{j}=\operatorname{deg}\left(f_{j}\right)$. Do likewise for $I(Z)_{t}$; it is easy to see that we get $\gamma_{t}=f_{1}^{c_{1}^{\prime}} \cdots f_{r}^{c_{r}^{\prime}}$ where $c_{j}^{\prime} \leq c_{j}$ for all $j$. Doing the same for $I(Z)_{t+1}$ gives $\gamma_{t+1}=f_{1}^{c_{1}^{\prime \prime}} \cdots f_{r}^{c_{r}^{\prime \prime}}$ with $c_{j}^{\prime \prime} \leq c_{j}^{\prime}$. Let $C_{j}$ be the normalization of the curve defined by $f_{j}$. The following theorem refines ideas of Fitchett [F1], [F2].

Theorem 1.2.1. Let $Z=m_{1} P_{1}+\cdots+m_{n} P_{n}$ for nonnegative integers $m_{i}$ and general points $P_{i} \in \mathbf{P}^{2}$. Let $t>\alpha(Z)$ with $c_{j}, c_{j}^{\prime}$ and $c_{j}^{\prime \prime}$ defined as above. If the SHGH Conjecture holds, then

$$
\begin{aligned}
\operatorname{dim} \operatorname{cok} \mu_{t} & \leq \sum_{j} d_{j}\left(c_{j}^{\prime}-c_{j}^{\prime \prime}\right)-\sum_{j}\left(\begin{array}{c}
c_{j}^{\prime}-c_{j}^{\prime \prime} \\
2
\end{array}\right) \\
+ & \sum_{j}\left(\left(\begin{array}{c}
c_{j}-c_{j}^{\prime}-a_{C_{j}} \\
2
\end{array}\right)+\left(\begin{array}{c}
c_{j}-c_{j}^{\prime}-b_{C_{j}} \\
2
\end{array}\right)\right) .
\end{aligned}
$$

In fact, the full SHGH Conjecture is not required for this theorem. One just needs that $I(Z)$ behaves as expected for the specific $Z$ being considered. We also propose the following conjecture:

\section{Conjecture 1.2.2. Equality holds in Theorem 1.2.1.}

As we show by examples in the appendix, the SHGH Conjecture allows one to determine conjectural values for the exponents $c_{j}, c_{j}^{\prime}$ and $c_{j}^{\prime \prime}$. As we show below, in many cases the splitting type of the curves $C_{j}$ is also known, and even in those cases where the type is not known, it is much easier to compute the splitting type of each $C_{j}$ symbolically than it is to compute $\operatorname{dim}$ cok $\mu_{t}$ symbolically in the usual way of finding a Gröbner basis of the ideal $I(Z)$. Thus the SHGH Conjecture and Conjecture 1.2.2. if true, allow one to determine the minimal number of homogeneous generators of $I(Z)$ in every degree except possibly degree $\alpha(Z)+1$. Examples in the appendix show how this is done.

We now put this into the context of minimal free graded resolutions. The minimal free graded resolution of $I(Z)$ is an exact sequence of the form $0 \rightarrow M_{1} \rightarrow M_{0} \rightarrow$ $I(Z) \rightarrow 0$, where $M_{0}$ (the module of generators) and $M_{1}$ (the module of syzygies) are free graded $R$-modules, hence of the form $M_{0}=\bigoplus_{i \geq 0} R[-i]^{g_{i}(Z)}$, and $M_{1}=$ $\bigoplus_{j \geq 0} R[-j]^{s_{j}(Z)}$ for nonnegative integers $g_{i}(Z)$ and $s_{j}(Z)$. (By $R[-i]$ we just mean the free $R$-module of rank 1 with the grading such that $R[-i]_{t}=R_{t-i}$.) The graded Betti numbers of $I(Z)$ are the sequences of integers $g_{i}(Z)$ and $s_{j}(Z)$ (which we write as $g_{i}$ and $s_{j}$ if $Z$ is understood). The Betti number $g_{i}$ is just dim cok $\mu_{i-1}$; 
hence $g_{i}$ is the number of generators of degree $i$ in any minimal set of homogeneous generators of $I(Z)$. Moreover, it is not hard to show that $g_{i}-s_{i}=\Delta^{3} h_{Z}(i)$ for all $i$, where $\Delta$ is the difference operator; i.e., $\Delta h_{Z}(i)=h_{Z}(i)-h_{Z}(i-1)$ (see p. 685 of $[\mathrm{FHH}]$.

1.3. The structure of the paper. We obtain our results by reformulating them in terms of complete linear systems on the surface $X$ obtained by blowing up $\mathbf{P}^{2}$ at the points $P_{1}, \ldots, P_{n}$.

In Section 2 we recall the background necessary for this reformulation, and we state known results needed for our approach. In Section 3 we state our main results and show how they lead to the statement in terms of fat points given above.

We also include an appendix for the purpose of showing how to obtain explicit predictions for the values of Hilbert functions of fat points and how our results lead to explicit numerical predictions for Betti numbers. In addition, Section A2 of the appendix discusses how to compute splitting types and discusses evidence in support of Conjecture 1.2.2. partly based on an approach for computing $g_{i}$ for $i>\alpha+1$ which is substantially more efficient than the usual methods, which involve finding a Gröbner basis of $I(Z)$. (A Macaulay 2 script which implements our method is included in Section A2.3 of the posted version of the paper, GHI2.) Even for relatively small values of the multiplicities $m_{i}$ and even for randomly chosen points $P_{i}$ over a finite field rather than for generic $P_{i}$, finding the graded Betti numbers $g_{i}$ for $I(Z)$ is beyond what can be done computationally by the usual methods. For example, we were unable to determine the graded Betti numbers of $I(Z)$ for $Z=77 P_{1}+\cdots+77 P_{7}+44 P_{8}+11 P_{9}+11 P_{10}+11 P_{11}$ by the usual methods, but our new computational method, based on the results of Section 3, working on an $800 \mathrm{MHz}$ computer over the finite field $|K|=31991$ using randomly chosen points $P_{i}$, determined the result in slightly over 5 minutes. The result, of course, is in agreement with our conjectural expected values. See also Example A1.2.3.

1.4. What was previously known. A lot of work has been done on the SHGH Conjecture. That the SHGH Conjecture holds for $n \leq 9$ points was known to Castelnuovo [Cas; a more modern proof is given by Nagata [N2]. The uniform case (i.e., $\left.Z=m\left(P_{1}+\cdots+P_{n}\right)\right)$ was proved for $m=2$ by [AC] (also see [P], which by the methods of [Ia] applies to the $m=2$ case), for $m=3$ by [Hi2], for $m \leq 12$ by CM2 and for $m \leq 20$ by [CCMO]. The case that $n$ is a square has likewise seen progressive improvements, with the main difficulty being to show $I(Z)_{t}=0$ when it is expected to be. For example, by specializing $n=16$ points to a smooth curve of degree 4 , it is not hard to show that $h_{Z}(t)$ has its expected value of $\left(\begin{array}{c}t+2 \\ 2\end{array}\right)-4\left(\begin{array}{c}m+1 \\ 2\end{array}\right)$ for all $t \geq 4 m+1$, while $h_{Z}(t)$ has its expected value of 0 for $t<4 m+1$ by [N1] for all $m$. A generalization of this in [HHF] shows that the SHGH Conjecture holds in all degrees $t$ such that $e\left(h_{z}, t\right)>0$ if $n$ is any square as long as $m$ is not too small. By more technical arguments one can show that the SHGH Conjecture holds also for small $m$ and $t$. For example, if $n$ is a power of 4, [E1] showed SHGH holds in the uniform case; $[\mathrm{BZ}$ extended this to $n$ being a product of powers of 4 and 9 ; and [HR] showed that the SHGH Conjecture holds for infinitely many $m$ for each square $n$. By [E2] it is now known to hold in the uniform case for any $m$ when $n$ is a square; alternate proofs have been given by [CM3] and [R].

Results for the general case (i.e., such that the multiplicities $m_{i}$ of $Z=\sum_{i} m_{i} P_{i}$ need not all be equal) are not as comprehensive. That the SHGH Conjecture holds 
in the case that $m_{i} \leq 3$ for all $i$ is due to CM1, improved to $m_{i} \leq 4$ by Mi] and then to $m_{i} \leq 7$ by $[\mathrm{Y}]$.

Previous results on graded Betti numbers seem to start with [Cat, which obtained a complete answer for $n \leq 5$ general points. This was extended to $n=6$ by [F3, then 7 by [Ha1] and 8 by [FHH]. For $n>8$, almost all results (and even conjectures) are for cases which are either uniform or close to uniform. For example, conjectures in the uniform case were put forward by Ha2, and in cases close to uniform by $\mathrm{HHF}$. (Those conjectures are consistent with Conjecture 1.2.2 due to Corollary 2.3.2.) The Betti numbers in the case of $n$ general points of multiplicity $m=1$ were determined by GGR. The uniform Betti numbers conjecture of [Ha2 was verified for $m=2$ by [I] and for $m=3$ by [GI]. More generally, if $Z=m_{1} P_{1}+\cdots+m_{n} P_{n}$, where the points $P_{i} \in \mathbf{P}^{2}$ are general and $m_{i} \leq 3$ for all $i$, then [BI] determines the graded Betti numbers in all degrees. By Theorem 3.2 of $\mathrm{HHF}$, applying E2, it follows that the uniform Betti numbers conjecture of Ha2 holds for all $m \geq(\sqrt{n}-2) / 4$ when the number $n$ of points is an even square. Additional cases are shown in [HR] when $n$ is not a square.

\section{BACKGROUND}

In this section we set notation and cite well-known facts which we will refer to later in the paper. Let $P_{1}, \ldots, P_{n}$ be distinct (not necessarily general) points of the projective plane $\mathbf{P}^{2}$. Let $p: X \rightarrow \mathbf{P}^{2}$ be the birational morphism given by blowing up the points.

2.1. Preliminaries. The divisor class group $\mathrm{Cl}(X)$ of divisors on $X$ modulo linear equivalence is the free abelian group with basis $L, E_{1}, \ldots, E_{n}$, where $E_{i}$ is the class of the divisor $p^{-1}\left(P_{i}\right)$ and $L$ is the pullback of the class of a line. Given any divisor $F$ on $X$, the dimension $h^{0}\left(X, \mathcal{O}_{X}(F)\right)$ of the global sections of $\mathcal{O}_{X}(F)$ depends only on the class $[F]$ of $F$. For convenience, we will denote $h^{0}\left(X, \mathcal{O}_{X}(F)\right)$ by either $h^{0}(X, F)$ or $h^{0}(X,[F])$, or even $h^{0}(F)$ or $h^{0}([F])$ if $X$ is understood.

Given any $F=t L-m_{1} E_{1}-\cdots-m_{n} E_{n}$, by Riemann-Roch we have

$$
h^{0}(X, F)-h^{1}(X, F)+h^{2}(F, X)=\frac{F^{2}-K_{X} \cdot F}{2}+1,
$$

where $K_{X}=-3 L+E_{1}+\cdots+E_{n}$ is the canonical class. Since $E_{i}$ is reduced and irreducible and $m_{i}=E_{i} \cdot F$, we have a canonical isomorphism $H^{0}\left(X, t L-\sum_{m_{i}>0} m_{i} E_{i}\right) \rightarrow H^{0}(X, F)$. (The idea is that if $|F|$ is nonempty, then $-\sum_{m_{i}<0} m_{i} E_{i}$ is contained in the base locus of $|F|$, essentially by Bézout's Theorem, and so $\left.|F|=\left(-\sum_{m_{i}<0} m_{i} E_{i}\right)+\left|t L-\sum_{m_{i}>0} m_{i} E_{i}\right|.\right) \quad$ On the other hand, $L$ is nef (meaning that $L \cdot C \geq 0$ for any effective divisor $C$ on $X$ ), so $h^{0}(X, F)=0$ if $t<0$. By duality we have $h^{2}(X, F)=h^{0}\left(X, K_{X}-F\right)$, so it follows that $h^{2}(X, F)=0$ whenever $t \geq 0$ (in fact, whenever $t \geq-2$ ).

If $t \geq 0$ and $m_{i} \geq 0$ for all $i$, then $\frac{F^{2}-K_{X} \cdot F}{2}+1=\left(\begin{array}{c}t+2 \\ 2\end{array}\right)-\sum_{i}\left(\begin{array}{c}m_{i}+1 \\ 2\end{array}\right)$, so Riemann-Roch gives

$$
h^{0}(X, F) \geq \max \left(0,\left(\begin{array}{c}
t+2 \\
2
\end{array}\right)-\sum_{i}\left(\begin{array}{c}
m_{i}+1 \\
2
\end{array}\right)\right) .
$$

This is just a manifestation of the canonical identification $H^{0}\left(X, t L-m_{1} E_{1}-\cdots-\right.$ $\left.m_{n} E_{n}\right)=H^{0}\left(\mathbf{P}^{2}, \mathcal{I}_{Z}(t)\right)=I(Z)_{t}$, where $Z=m_{1} P_{1}+\cdots+m_{n} P_{n}$ and $\mathcal{I}_{Z}$ is the sheaf of ideals defining $Z$. Because of this, given any integer $t$ and a fat point 
scheme $Z=m_{1} P_{1}+\cdots+m_{n} P_{n}$, we define $F_{t}(Z)=t L-m_{1} E_{1}-\cdots-m_{n} E_{n}$, and hence we have $h_{Z}(t)=h^{0}\left(X, F_{t}(Z)\right)$ for all $t$.

Given a divisor $F$ on $X$, let $\mu_{F}: H^{0}(X, F) \otimes H^{0}(X, L) \rightarrow H^{0}(X, F+L)$ denote the obvious natural map. By identifying $H^{0}\left(X, F_{t}(Z)\right)$ with $I(Z)_{t}$ and $H^{0}(X, L)$ with $H^{0}\left(\mathbf{P}^{2}, \mathcal{O}_{\mathbf{P}^{2}}(1)\right)=R_{1}$, it follows that $\operatorname{dim} \operatorname{cok} \mu_{t}(Z)=\operatorname{dim} \operatorname{cok} \mu_{F_{t}(Z)}$. Given a sum $F=H+N$ of effective divisors such that $|F|=N+|H|$ (i.e., such that $N$ is contained in the scheme theoretic base locus of $F$ ), Lemma 2.1.1 gives a simple but useful fact which relates $\operatorname{dim} \operatorname{cok} \mu_{F}$ to $\operatorname{dim} \operatorname{cok} \mu_{H}$. (For the proof, observe that $N$ is in the base locus of the image $\operatorname{Im} \mu_{F}$ of $\mu_{F}$, i.e., that $\operatorname{Im} \mu_{F}=N+\operatorname{Im} \mu_{H}$. See Lemma 2.10(b) of Ha3.) Obviously $\mu_{F}$ is injective when $F$ is not effective, and, when $F$ is effective, $F$ decomposes as in Lemma 2.1.1. Thus Lemma 2.1.1 reduces the general problem of computing $\operatorname{dim} \operatorname{cok} \mu_{F}$ to the case that $|F|$ is effective and fixed component free, and thus in particular to the case that $F$ is nef.

Lemma 2.1.1. Let $F=H+N$ be a sum of effective divisors $H$ and $N$ on the surface $X$ such that $|F|=N+|H|$. Then

$$
\operatorname{dim} \operatorname{cok} \mu_{F}=\left(\operatorname{dim} \operatorname{cok} \mu_{H}\right)+\left(h^{0}(X, F+L)-h^{0}(X, H+L)\right) .
$$

It is easy to give examples such that $F=N$ (and hence $\mu_{F}$ is injective) but that $N+|L|$ is a proper subset of $|F+L|$ (and hence the map $\mu_{F}$ cannot be surjective). For example, if $C$ is an exceptional curve (i.e., $C$ is smooth and rational with $\left.C^{2}=-1\right)$ with $d=C \cdot L$, then the kernel of $\mu_{L+(d+1) C}$ is nonzero since already $\mu_{L}$ has nonzero kernel, and $\mu_{L+(d+1) C}$ is not onto since $C$ is in the base locus of $|L+(d+1) C|$ but $|2 L+(d+1) C|$ is base curve free. In particular, the occurrence of fixed components is one reason that $\mu_{F}$ can fail to have maximal rank (i.e., fail to be either injective or surjective). What motivates this paper is that this is not the only reason. In fact, $\mu_{F}$ can fail to have maximal rank even when $F$ is very ample. (For example, let $F=\left(3 L-E_{1}-\cdots-E_{7}\right)+m\left(8 L-3 E_{1}-\cdots-3 E_{7}-E_{8}\right)$, where the points $P_{i}$ are general and $m \geq 1$. Then $F$ is very ample [Ha5, but $\mu_{F}$ fails to have maximal rank [FHH].) The point of this paper is that, when $F-L$ is effective, the failure of $\mu_{F}$ to have maximal rank depends on the fixed components of $|F-L|$.

Note that if $C$ is a plane curve contributory for points $P_{i}$, then $C^{\prime}$ is an exceptional curve, where $C^{\prime}$ is the proper transform of $C$ on the surface $X$ obtained by blowing up the points $P_{i}$. As discussed in the introduction, our geometric approach for determining the dimension of the cokernel of $\mu_{F}$ for certain divisors $F$ thus depends on knowing the splitting $\mathcal{O}_{E}\left(-a_{E}\right) \oplus \mathcal{O}_{E}\left(-b_{E}\right)$ of the restriction $\left.p^{*} \Omega(1)\right|_{E}$ of $p^{*} \Omega(1)$ to exceptional curves $E$. The following result (see [As, or [F1], [F2]) covers most of what is known:

Lemma 2.1.2. Let $E \subset X$ be a smooth rational curve, where $p: X \rightarrow \mathbf{P}^{2}$ is the morphism blowing up distinct points $P_{i}$ of $\mathbf{P}^{2}$. Let $d=E \cdot L$ and let $m$ be the maximum of $E \cdot E_{i}, 1 \leq i \leq n$. Then there are integers $0 \leq a_{E} \leq b_{E} \leq d$ with $\min (m, d-m) \leq a_{E} \leq d-m$ and $d=a_{E}+b_{E}$ such that $\left.\left(p^{*} \Omega_{\mathbf{P}^{2}}(1)\right)\right|_{E}$ is isomorphic to $\mathcal{O}_{E}\left(-a_{E}\right) \oplus \mathcal{O}_{E}\left(-b_{E}\right)$.

Note that, if $d \leq 2 m+1$, then $a_{E}=\min (m, d-m)$ and $b_{E}=\max (m, d-m)$. For cases not covered by Lemma 2.1.2 $a_{E}$ and $b_{E}$ can be computed fairly efficiently. We will describe an algorithm for doing so in Section $\mathrm{A2}$ of the appendix. 
2.2. The SHGH conjecture. Here we state the version of the SHGH Conjecture given in [Ha4. This version is simple to state and useful conceptually. We include in the appendix an equivalent version that is more useful for obtaining explicit conjectural values of Hilbert functions.

Conjecture 2.2.1. Let $X$ be a surface obtained by blowing up $n$ generic points of $\mathbf{P}^{2}$. Then every reduced irreducible curve $C \subset X$ with $C^{2}<0$ is an exceptional curve and either $h^{0}(X, F)=0$ or $h^{1}(X, F)=0$ for every nef divisor $F$ on $X$.

Theorem 2.2.2. Conjecture 2.2.1 implies Conjectures 1.1.1 and 1.1.2.

Proof. First, note that if $H$ is effective, then $h^{2}(X, H)=0$ by duality (since $L$ is nef but $\left.\left(K_{X}-H\right) \cdot L<0\right)$. Now let $C$ be nef and effective. Then $H+C$ is also nef and effective. By Conjecture 2.2.1, $h^{1}(X, H)=0=h^{1}(X, H+C)$ and $h^{1}(X, C)=0=h^{2}(X, C)$ (hence $\left(C^{2}-C \cdot K_{X}\right) / 2 \geq 0$ by Riemann-Roch). Assume $H \cdot C>0$. Applying Riemann-Roch for surfaces now gives $h^{0}(X, H+C)=$ $h^{0}(X, H)+\left(C^{2}-C \cdot K_{X}\right) / 2+H \cdot C>h^{0}(X, H)$. Similarly, if $C$ is instead an exceptional curve with $C \cdot H>0$, then $H+C$ is nef and effective, and $h^{0}(X, H+C)>$ $h^{0}(X, H)$. In particular, if $H$ is nef and effective with $|H|$ base curve free, and if $C$ is a prime divisor which is a base curve of $|H+C|$ such that $C$ is either exceptional or $C^{2}>0$ (hence nef), then $C \cdot H=0$.

Now consider $Z=m_{1} P_{1}+\cdots+m_{n} P_{n}$ and let $\alpha=\alpha(Z)$. Then $h_{Z}(t)=h^{0}(X, F)$, where $F=t L-m_{1} E_{1}-\cdots-m_{n} E_{n}$. Let $H$ be the moving part of $|F|$, and decompose the fixed part as $D+N$, where $D$ is the sum of the curves in the base locus of $|F|$ of nonnegative self-intersection and $N$ is the sum of the curves in the base locus of negative self-intersection. By Conjecture 2.2.1 each curve in $N$ is an exceptional curve, hence disjoint from $H$ and $D$. In addition, these exceptional curves are pairwise orthogonal (since if $C$ and $C^{\prime}$ both appear in $N$ and have $C \cdot C^{\prime}>0$, then $h^{0}\left(X, C+C^{\prime}\right) \geq C \cdot C^{\prime}+1$ by Riemann-Roch; hence $C+C^{\prime}$ cannot be part of the base locus of $\left.|F|\right)$. Also, none of the exceptional curves $E$ appearing in $N$ is the blowup of a point $P_{i}$ (since if it were we would have $\left.m_{i}=E \cdot F=E \cdot(H+D+N)=E \cdot N<0\right)$. Thus for some $c_{j_{k}}$ we have $N=\sum_{k} c_{j_{k}} C_{j_{k}}$, where $C_{j}$ is the proper transform of the plane curve defined by $f_{j}$ in the statement of Conjecture 1.1.2. Applying Riemann-Roch gives

$$
\begin{aligned}
h_{Z}(t) & =h^{0}(X, F)=h^{0}(X, F-N)=\left((F-N)^{2}-K_{X} \cdot(F-N)\right) / 2+1 \\
& =\left(F^{2}-K_{X} F\right) / 2+1+\sum_{k}\left(\begin{array}{c}
c_{j_{k}} \\
2
\end{array}\right)=\left(\begin{array}{c}
t+2 \\
2
\end{array}\right)-\sum_{i}\left(\begin{array}{c}
m_{i}+1 \\
2
\end{array}\right)+\sum_{k}\left(\begin{array}{c}
c_{j_{k}} \\
2
\end{array}\right),
\end{aligned}
$$

as claimed in Conjecture 1.1 .2

Now consider degree $t+1$, so $t+1>\alpha(Z)$. Then $h_{Z}(t+1)=h^{0}(X, F+L)$, where $F+L=H+(L+D)+N$. Note that, as we saw above, $|L+D|$ is fixed component free. Thus the fixed part of $|F+L|$ consists at most of exceptional curves coming from $N=\sum_{k} c_{j_{k}} C_{j_{k}}$. In fact, by the first paragraph of the proof, $\left|F+D+L+\sum_{k} \min \left(d_{j_{k}}, c_{j_{k}}\right) C_{j_{k}}\right|$ is base curve free and $|F+D+L+N|=\left|F+D+L+\sum_{k} \min \left(d_{j_{k}}, c_{j_{k}}\right) C_{j_{k}}\right|+\sum_{k} \max \left(0, c_{j_{k}}-d_{j_{k}}\right) C_{j_{k}}$. I.e., $\sum_{k} \max \left(0, c_{j_{k}}-d_{j_{k}}\right) C_{j_{k}}$ is the divisorial part of the base locus of $|F+L|$. If we denote $\max \left(0, c_{j_{k}}-d_{j_{k}}\right)$ by $b_{j_{k}}$ and reindex, this becomes $\sum_{l} b_{l} C_{l}$, and applying Riemann-Roch as above gives

$$
h_{Z}(t+1)=h^{0}(X, F+L)=\left(\begin{array}{c}
(t+1)+2 \\
2
\end{array}\right)-\sum_{i}\left(\begin{array}{c}
m_{i}+1 \\
2
\end{array}\right)+\sum_{l}\left(\begin{array}{c}
b_{l} \\
2
\end{array}\right)
$$


as claimed in Conjecture 1.1.1. (Note that $t+1$ here is the same as $t$ in the statement of Conjecture 1.1.1, since there we assumed $t>\alpha(Z)$, but here, in order to handle Conjecture 1.1 .2 simultaneously, we assumed only $t \geq \alpha(Z)$.)

2.3. Mumford's snake lemma. Mumford [Mu1] applied the snake lemma to questions related to $\mu_{F}$. We recall that now. To do so we establish some notation that we will use here and throughout the paper. Let $F, C$ and $D$ be divisors on $X$ with $C$ effective; then we have the natural multiplication maps

$$
\mu_{F, D}: H^{0}(X, F) \otimes H^{0}(X, D) \rightarrow H^{0}(X, F+D)
$$

and

$$
\mu_{C ; F, D}: H^{0}\left(C,\left.F\right|_{C}\right) \otimes H^{0}(X, D) \rightarrow H^{0}\left(C,\left.(F+D)\right|_{C}\right) .
$$

In the particular case that $D=L$, which is almost always true in the present paper, we write $\mu_{F}$ and $\mu_{C ; F}$ instead of $\mu_{F, L}$ and $\mu_{C ; F, L}$.

Lemma 2.3.1. Let $p: X \rightarrow \mathbf{P}^{2}$ be a blowup of $\mathbf{P}^{2}$ at $n$ distinct points with $L, E_{1}, \ldots, E_{n}$ as usual. Let $D$ be a divisor on $X$, let $V=H^{0}(X, D)$, and let $F$ and $C$ be divisors on $X$ with $C$ effective and with $h^{1}(X, F)=0=h^{1}(X, F+D)$. Then the following diagram is commutative with exact rows:

(०) $0 \longrightarrow H^{0}(X, F) \otimes V \longrightarrow H^{0}(X, F+C) \otimes V \longrightarrow H^{0}\left(C,\left.(F+C)\right|_{C}\right) \otimes V \longrightarrow 0$

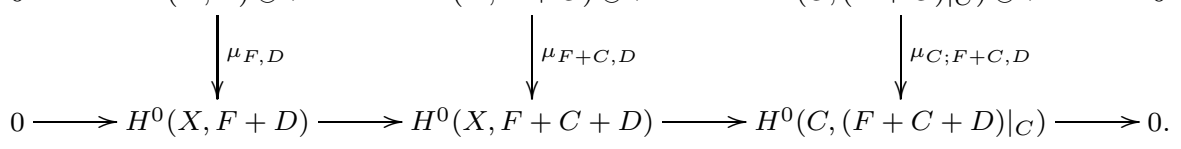

The snake lemma thus gives an exact sequence

$$
\begin{aligned}
0 & \rightarrow \operatorname{ker} \mu_{F, D} \rightarrow \operatorname{ker} \mu_{F+C, D} \rightarrow \operatorname{ker} \mu_{C ; F+C, D} \\
& \rightarrow \operatorname{cok} \mu_{F, D} \rightarrow \operatorname{cok} \mu_{F+C, D} \rightarrow \operatorname{cok} \mu_{C ; F+C, D} \rightarrow 0,
\end{aligned}
$$

which we will refer to as $\mathcal{S}(F, C, D)($ or $\mathcal{S}(F, C)$ if $D=L)$.

Another useful fact is the Castelnuovo-Mumford Lemma Mu2, which gives a criterion for $\mu_{F}$ to not only have maximal rank but to be surjective. The version we state, Corollary 2.3.2, follows easily from Lemma 2.3.1 using $\mathcal{S}(0, L, H)$. (The hypothesis $h^{1}(X, H-L)=0$ is used to ensure that $H^{0}(X, H) \rightarrow H^{0}\left(L,\left.H\right|_{L}\right)$ is surjective; $H \cdot L \geq 0$ then ensures that $\operatorname{cok} \mu_{L ; L, H}=0$.)

Corollary 2.3.2. Let $p: X \rightarrow \mathbf{P}^{2}$ be obtained by blowing up $n$ distinct points of $\mathbf{P}^{2}$, with $L$ the pullback of the class of a line. If $H$ is a divisor on $X$ with $h^{1}(X, H-L)=0$ and $H \cdot L \geq 0$, then $\operatorname{cok} \mu_{H}=0$.

When $D=L$, explicit expressions for the kernels and cokernels in Lemma 2.3.1 can be given in terms of the cotangent bundle. Recall the Euler sequence defining $\Omega=\Omega_{\mathbf{P}^{2}}$ :

$$
0 \rightarrow \Omega(1) \rightarrow \mathcal{O}_{\mathbf{P}^{2}} \otimes H^{0}\left(\mathbf{P}^{2}, \mathcal{O}_{\mathbf{P}^{2}}(1)\right) \rightarrow \mathcal{O}_{\mathbf{P}^{2}}(1) \rightarrow 0 .
$$

Pulling the Euler sequence back to $X$, tensoring by $\mathcal{O}_{X}(F)$ and identifying $H^{0}\left(\mathbf{P}^{2}, \mathcal{O}_{\mathbf{P}^{2}}(1)\right)$ with $H^{0}(X, L)$ gives an exact sequence

$$
0 \rightarrow\left(p^{*} \Omega\right)(F+L) \rightarrow \mathcal{O}_{X}(F) \otimes H^{0}(X, L) \rightarrow \mathcal{O}_{X}(F+L) \rightarrow 0 .
$$

If $h^{1}(X, F)=0$, then taking cohomology gives an exact sequence

$$
\begin{aligned}
0 \rightarrow H^{0}\left(X,\left(p^{*} \Omega\right)(F+L)\right) \rightarrow H^{0}(X, F) \otimes H^{0}(X, L) & \rightarrow H^{0}(X, F+L) \\
& \rightarrow H^{1}\left(X,\left(p^{*} \Omega\right)(F+L)\right) \rightarrow 0 ;
\end{aligned}
$$


hence ker $\mu_{F}=H^{0}\left(X,\left(p^{*} \Omega\right)(F+L)\right)$ and cok $\mu_{F}=H^{1}\left(X,\left(p^{*} \Omega\right)(F+L)\right)$. Similarly, if $h^{1}\left(C,\left.F\right|_{C}\right)=0$, by restricting ( $\left.\dagger\right)$ to $C$ and taking cohomology we get ker $\mu_{C ; F}=$ $H^{0}\left(C,\left.\left(\left(p^{*} \Omega\right)(F+L)\right)\right|_{C}\right)$ and $\operatorname{cok} \mu_{C ; F}=H^{1}\left(C,\left.\left(\left(p^{*} \Omega\right)(F+L)\right)\right|_{C}\right)$. In case $C$ is a smooth rational curve, taking $t=F \cdot C$ we have:

$$
\begin{aligned}
& \operatorname{ker} \mu_{C ; F}=H^{0}\left(C, \mathcal{O}_{C}\left(t-a_{C}\right) \oplus \mathcal{O}_{C}\left(t-b_{C}\right)\right), \\
& \operatorname{cok} \mu_{C ; F}=H^{1}\left(C, \mathcal{O}_{C}\left(t-a_{C}\right) \oplus \mathcal{O}_{C}\left(t-b_{C}\right)\right)
\end{aligned}
$$

with $a_{C}$ and $b_{C}$ as defined in Section 1.2 .

\section{MAin RESUlts}

Given a divisor $F$ on a blowup $X$ of $\mathbf{P}^{2}$ at $n$ general points, the naive conjecture that $h^{0}(X, F)$ always equals $\max \left(0,\left(F^{2}-K_{X} \cdot F\right) / 2+1\right)$ is false. One way to salvage it is to impose a niceness requirement on $F$, such as to require that $F$ be nef, or even that $F \cdot C \geq 0$ for all exceptional $C$, which is weaker. In fact, an equivalent version of the SHGH Conjecture is given by Conjecture A1.2.1, which just conjectures that $h^{0}(X, F)=\max \left(0,\left(F^{2}-K_{X} \cdot F\right) / 2+1\right)$ whenever $F \cdot C \geq 0$ for all exceptional $C$.

Likewise, the naive conjecture that $\mu_{F}$ should always have maximal rank is also false, as we saw above. Again we can try to salvage the naive conjecture by imposing a niceness requirement on $F$, but the necessary requirement is more subtle. Assuming we can compute $h^{0}(X, F)$ for an arbitrary divisor $F$, Lemma 2.1.1 reduces the problem of computing the rank of $\mu_{F}$ in general to the case that $F$ is effective and fixed component free. But, as we mentioned above, even if $F$ is effective and fixed component free, or even very ample, $\mu_{F}$ can fail to have maximal rank.

Instead, we will consider all $F \in L+\operatorname{EFF}(X)$, where $\operatorname{EFF}(X)$ is the subsemigroup of the divisor class group of $X$ of classes of all effective divisors. In this section, refining ideas of Fitchett, we will give an upper bound on the dimension of the cokernel of $\mu_{F}$ for certain $F \in L+\operatorname{EFF}(X)$ (for all of them if the SHGH Conjecture is true). We conjecture that this upper bound is in fact an equality.

Assuming the SHGH Conjecture, Fitchett reduced the problem of handling $\mu_{F}$ for an arbitrary $F \in L+\operatorname{EFF}(X)$ to the case $F=L+m E$ where $E$ is an exceptional curve and $0 \leq m \leq L \cdot E$. (See [F1, [F2, which give explicit bounds on $\operatorname{dim} \operatorname{cok} \mu_{F}$ for $F \in L+\mathrm{EFF}$ in the case of $n \leq 8$ general points, using a construction originally described in Fitchett's thesis.)

We now recall Fitchett's idea, assuming that $X$ is obtained by blowing up $n$ general points. Note that the SHGH Conjecture would make the assumption $h^{1}(X, H)=0$ automatic.

Proposition 3.1. Let $F \in L+E F F(X)$, so we have the decomposition $F-L=$ $H+N$ given by Lemma A1.1.1 (d,e), where $H$ is effective with $H \cdot E \geq 0$ for all exceptional $E$ and where either $N=0$ or $N=c_{1} C_{1}+\cdots+c_{r} C_{r}$ for some mutually disjoint exceptional curves $C_{i}$ and integers $c_{i}>0$. Assume that $h^{1}(X, H)=0$. Then, $\operatorname{cok} \mu_{F} \cong \bigoplus_{i=1, \ldots, r} \operatorname{cok} \mu_{L+c_{i} C_{i}} \cong \operatorname{cok} \mu_{L+N}$. If moreover $F \cdot C_{i} \geq 0$, then $0<c_{i} \leq L \cdot C_{i}$.

Proof. By Lemma A1.1.1(d,e), $F=L+H+N$ where either $N=0$ or $N=$ $c_{1} C_{1}+\cdots+c_{r} C_{r}$ for some mutually disjoint exceptional curves $C_{i}$ and integers $c_{i}>0$, and where $H$ is effective and orthogonal to $N$. Then $h^{1}(X, H)=0$ implies 
$\operatorname{cok} \mu_{L+H}=0$ by Corollary 2.3.2. Taking the cohomology of

$$
0 \rightarrow \mathcal{O}_{X}(H) \rightarrow \mathcal{O}_{X}(L+H) \rightarrow \mathcal{O}_{L}(H+L) \rightarrow 0
$$

and using $h^{1}(X, H)=0$ implies that $h^{1}(X, L+H)=0$. Similarly, we also have $h^{1}(X, 2 L+H)=0$. So the sequence $\mathcal{S}(F-N, N)$ of Lemma 2.3.1 holds (since $F-N=L+H)$ and tells us that the cokernels for $\mu_{F}$ and $\mu_{N ; F}$ are isomorphic. But $\mathcal{O}_{N}(F)$ is isomorphic to $\mathcal{O}_{N}(L+N)$, since $H \cdot N=0$, and the $C_{i}$ are disjoint, so $\mathcal{O}_{N}(L+N) \cong \bigoplus \mathcal{O}_{c_{i} C_{i}}\left(L+c_{i} C_{i}\right)$; we finally get that the cokernel of $\mu_{N ; F}$ is isomorphic to the direct sum of the cokernels of $\mu_{c_{i} C_{i} ; L+c_{i} C_{i}}$. Moreover, $h^{1}(X, L)=$ $h^{1}(X, 2 L)=0$, so the sequence $\mathcal{S}\left(L, c_{i} C_{i}\right)$ of Lemma 2.3.1 gives $\operatorname{cok} \mu_{c_{i} C_{i} ; L+c_{i} C_{i}} \cong$ $\operatorname{cok} \mu_{L+c_{i} C_{i}}$ since $\operatorname{cok} \mu_{L}=0$. Hence, as Fitchett observed, the cokernel for $\mu_{F}$ is isomorphic to the direct sum of the cokernels for $\mu_{L+c_{i} C_{i}}$. Running the same argument with $L+N$ in place of $F$ now gives $\bigoplus_{i=1, \ldots, r} \operatorname{cok} \mu_{L+c_{i} C_{i}} \cong \mu_{L+N}$.

This and Lemma 2.1.1 motivate the following problem:

Problem 3.2. Determine the rank of $\mu_{F}$ for each $F=L+i E$, where $E$ is smooth and rational with $E^{2}=-1$ and $0 \leq i \leq E \cdot L$.

Concerning this problem, we prove Theorem 3.3, which gives explicit upper bounds for the dimension of $\operatorname{cok} \mu_{L+i E}$. Similar but less precise results were given in [F1], F2]. Theorem 3.3(c) seems to be entirely new, however.

One can also give a lower bound for $\operatorname{dim} \operatorname{cok} \mu_{F}$. This lower bound, as is the case for the upper bound given in Theorem 3.3. is just what one can conclude from the sequence below of short exact sequences $(\ddagger)$. An explicit formula for this lower bound turns out to be more complicated and less useful than that for the upper bound, so we do not include it here.

Theorem 3.3. Let $X$ be the blowup of $\mathbf{P}^{2}$ at $n$ distinct points $P_{1}, \ldots, P_{n}$, and take $L, E_{1}, \ldots, E_{n}$ as usual. Let $F=L+i E$, where $d=E \cdot L, m$ is the maximum of $E \cdot E_{j}$ over $1 \leq j \leq n$, and where $E$ is smooth and rational with $E^{2}=-1$ and $0 \leq i \leq d$. Then we have

$$
\operatorname{dim} \operatorname{cok} \mu_{F} \leq\left(\begin{array}{c}
i-b_{E} \\
2
\end{array}\right)+\left(\begin{array}{c}
i-a_{E} \\
2
\end{array}\right)
$$

with equality in the following cases:

(a) $i \leq a_{E}+2$;

(b) $b_{E}-a_{E} \leq 2$; or

(c) $a_{E}=d-m$.

Proof. Let $F_{j}=L+j E$ for $0 \leq j<i$; it is easy to check that $H^{1}\left(F_{j}\right)=0=$ $H^{1}\left(F_{j}+L\right)$, so we can consider $\mathcal{S}\left(F_{j}, E\right)$. Since $\left(F_{j}+E\right) \cdot E=d-j \geq 0$, by (††) and Lemma 2.1.2 we have

$$
\operatorname{ker} \mu_{E ; F_{j}}=H^{0}\left(E, \mathcal{O}_{E}\left(b_{E}-j\right)\right) \oplus H^{0}\left(E, \mathcal{O}_{C}\left(a_{E}-j\right)\right) \text {, }
$$

$\operatorname{cok} \mu_{E ; F_{j}}=H^{1}\left(E, \mathcal{O}_{E}\left(b_{E}-j\right)\right) \oplus H^{1}\left(E, \mathcal{O}_{E}\left(a_{E}-j\right)\right)$, and $\operatorname{dim} \operatorname{ker} \mu_{E ; F_{j}}=$ $\left(b_{E}-j+1\right)_{+}+\left(a_{E}-j+1\right)_{+}$, while $\operatorname{dim} \operatorname{cok} \mu_{E ; F_{j}}=\left(j-b_{E}-1\right)_{+}+\left(j-a_{E}-1\right)_{+}$. 
Writing $a$ and $b$ for $a_{E}$ and $b_{E}$, we have the following exact sequences:

$$
\begin{array}{lll}
\operatorname{cok} \mu_{F_{i-1}} & \rightarrow \operatorname{cok} \mu_{F_{i}} & \rightarrow H^{1}\left(\mathcal{O}_{E}(b-i) \oplus \mathcal{O}_{E}(a-i)\right) \rightarrow 0, \\
\operatorname{cok} \mu_{F_{i-2}} & \rightarrow \operatorname{cok} \mu_{F_{i-1}} & \rightarrow H^{1}\left(\mathcal{O}_{E}(b-i+1) \oplus \mathcal{O}_{E}(a-i+1)\right) \rightarrow 0, \\
\cdots & & \\
\operatorname{cok} \mu_{L+E} & \rightarrow \operatorname{cok} \mu_{L+2 E} & \rightarrow H^{1}\left(\mathcal{O}_{E}(b-2) \oplus \mathcal{O}_{E}(a-2)\right) \rightarrow 0, \\
\operatorname{cok} \mu_{L} \rightarrow \operatorname{cok} \mu_{L+E} & \rightarrow H^{1}\left(\mathcal{O}_{E}(b-1) \oplus H^{1}\left(\mathcal{O}_{E}(a-1)\right) \rightarrow 0 .\right.
\end{array}
$$

Note that $\operatorname{cok} \mu_{L}=0$; this is just the fact that $R_{1} \otimes R_{1}$ maps by multiplication surjectively to $R_{2}$, where $R$ is the ring $K\left[\mathbf{P}^{2}\right]$. Since $\operatorname{cok} \mu_{L}=0$, $\operatorname{dim} \operatorname{cok} \mu_{F_{i}}$ is at most the sum of the dimensions of the column of $H^{1}$ 's; i.e., we have $\operatorname{dim} \operatorname{cok} \mu_{F_{i}} \leq$ $\sum_{j \geq 0}\left((i-b-1-j)_{+}+(i-a-1-j)_{+}\right)=\left(\begin{array}{c}i-b \\ 2\end{array}\right)+\left(\begin{array}{c}i-a \\ 2\end{array}\right)$, and equality holds if and only if the displayed sequences are all exact on the left. Moreover, $h^{1}\left(\mathcal{O}_{E}(b-j) \oplus \mathcal{O}_{E}(a-j)\right)=0$ for all $j \leq a+1$, so cok $\mu_{F_{j}}=0$ for all $0 \leq j \leq a+1$. Thus each sequence is exact for which $F_{j}$ in the middle column has index $j \leq a+2$. This implies claim (a). Moreover, any of the sequences for which dim $\operatorname{ker} \mu_{E ; F_{j}}=0$ will also be exact on cokernels, and $\operatorname{dim} \operatorname{ker} \mu_{E ; F_{j}}=0$ for all $j \geq b+1$. It follows that equality holds if $j>a+2$ implies $j>b$, i.e., if $a+2 \geq b$. This shows (b).

Finally, consider (c); thus $b=m$. It is enough to show that the maps $\operatorname{ker} \mu_{F_{j}} \rightarrow$ ker $\mu_{E ; F_{j}}$ are onto for $a+3 \leq j \leq b$; we already observed above that exactness holds on cokernels (and hence for kernels) for other values of $j$. We may assume, after reindexing if need be, that $E_{1} \cdot E=m$.

From the exact sequence $0 \rightarrow \mathcal{O}_{E}(m-d) \rightarrow \mathcal{O}_{E} \otimes H^{0}\left(X, L-E_{1}\right) \rightarrow \mathcal{O}_{E}(d-m) \rightarrow 0$ we see that ker $\mu_{E ; F_{j}, L-E_{1}} \cong H^{0}\left(E, F_{j} \cdot E+m-d\right)=H^{0}(E, m-j)$. The inclusion $H^{0}\left(X, L-E_{1}\right) \subset H^{0}(X, L)$ induces an inclusion $\operatorname{ker} \mu_{E ; F_{j}, L-E_{1}} \rightarrow \operatorname{ker} \mu_{E ; F_{j}, L}=$ ker $\mu_{E ; F_{j}}$. The cokernel is isomorphic to $H^{0}\left(E, \mathcal{O}_{E}(a-j)\right)$ (see the exact sequence (4) in the proof of Theorem 3.1 of [F1]). Unwinding definitions, we see that the induced map $H^{0}(E, m-j) \cong \operatorname{ker} \mu_{E ; F_{j}, L-E_{1}} \hookrightarrow \operatorname{ker} \mu_{E ; F_{j}}$ sends an element $\sigma \in H^{0}(E, m-j)$ to $\left.x\right|_{E} \sigma \otimes y-\left.y\right|_{E} \sigma \otimes x \in \operatorname{ker} \mu_{E ; F_{j}}$ if we choose homogeneous coordinates $x, y$ and $z$ on $\mathbf{P}^{2}$ such that $P_{1}$ is the point where $x=0=y$.

Since $a+3 \leq j \leq b=m$, the induced inclusion $H^{0}(E, m-j) \hookrightarrow \operatorname{ker} \mu_{E ; F_{j}}$ is an isomorphism. Moreover, taking the cohomology of $0 \rightarrow \mathcal{O}_{X}\left(E_{1}+(j-1) E\right) \rightarrow$ $\mathcal{O}_{X}\left(E_{1}+j E\right) \rightarrow \mathcal{O}_{E}(m-j) \rightarrow 0$ gives the map $H^{0}\left(X, E_{1}+j E\right) \rightarrow$ $H^{0}\left(E, \mathcal{O}_{E}(m-j)\right)$, which is surjective for $1 \leq j \leq m+1$ since $h^{1}\left(E, \mathcal{O}_{E}(m-j)\right)$ is 0 in this range and, by induction on $j$ starting with $j=1$, so is $h^{1}\left(X, \mathcal{O}_{X}\left(E_{1}+(j-1) E\right)\right)$. Composing $H^{0}\left(X, E_{1}+j E\right) \rightarrow H^{0}\left(E, \mathcal{O}_{E}(m-j)\right)$ with the induced isomorphism $H^{0}(E, m-j) \rightarrow \operatorname{ker} \mu_{E ; F_{j}}$ gives for each $f \in$ $H^{0}\left(X, E_{1}+j E\right)$ the map $\left.\left.\left.f \mapsto f\right|_{E} \mapsto x\right|_{E} f\right|_{E} \otimes y-\left.\left.y\right|_{E} f\right|_{E} \otimes x \in \operatorname{ker} \mu_{E ; F_{j}}$. Thus every element of ker $\mu_{E ; F_{j}}$ is of the form $\left.\left.\left.f\right|_{E} \mapsto x\right|_{E} f\right|_{E} \otimes y-\left.\left.y\right|_{E} f\right|_{E} \otimes x$ where $f \in H^{0}\left(X, E_{1}+j E\right)$. But $\left.\left.\left.f\right|_{E} \mapsto x\right|_{E} f\right|_{E} \otimes y-\left.\left.y\right|_{E} f\right|_{E} \otimes x$ is the image of $x f \otimes y-y f \otimes x \in \operatorname{ker} \mu_{F_{j}}$ under the map $\operatorname{ker} \mu_{F_{j}} \rightarrow \operatorname{ker} \mu_{E ; F_{j}}$, so the map is surjective.

In fact, we do not know any times that the equality in the theorem does not hold. This suggests the following conjecture:

Conjecture 3.4. Let $F=L+i E$ be as in Theorem 3.3 for general points $P_{i}$. Then $\operatorname{dim} \operatorname{cok} \mu_{F}=\left(\begin{array}{c}i-b_{E} \\ 2\end{array}\right)+\left(\begin{array}{c}i-a_{E} \\ 2\end{array}\right)$.

Remark 3.5. (a) Conjecture 3.4 is equivalent to the first column of maps in $(\ddagger)$ all being injective. Hence if Conjecture 3.4 holds for some $i$, then it holds for all 
$0 \leq j \leq i$. Moreover, Conjecture 3.4 holds for $i=L \cdot E$ if and only if it holds for all $a_{E}+3 \leq i \leq b_{E}$, since the proof of Theorem 3.3 shows that the first column of maps in $(\ddagger)$ are injective for $i \leq a_{E}+2$ and for $i>b_{E}$.

(b) In the notation of Proposition 3.1, assuming the SHGH Conjecture and Conjecture 3.4. and assuming we can determine splitting types, we thus can determine the dimension of the cokernel of $\mu_{F}$ for any $F$ as long as $|F-L|$ is not empty. The splitting type of an exceptional curve can be computed fairly efficiently, at least provisionally (that is, by Macaulay 2 GS, say, in positive characteristic, using randomly chosen points; a Macaulay 2 script that does this is included in Section A2.3 of the posted version of this paper, GHI2]. We discuss this, and we give additional, computational, support for Conjecture 3.4 in Section A2 of the appendix.

(c) Translating in terms of fat points, this says that we can produce conjectural dimensions for the cokernels of $\mu_{t}$ for $I(Z)$ in every degree $t$ but $t=\alpha(Z)$. We can even sometimes determine the dimension for the cokernels of $\mu_{\alpha}$, for example by applying Lemma A1.2.6 (see Example A1.2.7), or if $h_{Z}(\alpha)=1$, or if $F_{\alpha}(Z)$ decomposes as $F_{\alpha}(Z)=L+H+N$, where $h^{1}(X, H)=0$ and $H \cdot L \geq 0$, even if $H$ is not effective (see Example A1.2.8).

(d) Conjecture 1.2 .2 is equivalent to Conjecture 3.4 assuming the SHGH Conjecture. The first sum in the bound in Theorem 1.2.1 is exactly the difference term in Lemma 2.1.1, which accounts for the contribution to the cokernel owing to loss in fixed components in going from degree $t$ to degree $t+1$. (This term does not occur in Conjecture 3.4 since the $F$ there is base curve free.) The second sum in the bound in Theorem 1.2.1 sums up exactly what each of the disjoint exceptional curves in the base locus of $F_{t}(Z)-L$ should contribute to the cokernel, according to Proposition 3.1 and Conjecture 3.4

Proof of Theorem 1.2.1. Let $t>\alpha(Z)$ and let $F=F_{t}(Z)$. Thus $F-L$ is effective. By the SHGH Conjecture (2.2.1), as in the proof of Theorem 2.2.2, we have $F-$ $L=H+N$, where $H$ is nef and effective with $h^{1}(X, H)=0$, and where $N=$ $c_{1} C_{1}+\cdots+c_{r} C_{r}$ is a sum of pairwise orthogonal exceptional curves orthogonal to $H$ with the curves $C_{j}$ being the proper transforms of those curves in the base locus of $I(Z)_{t-1}$ which are negative for the points $P_{i}$. Let $N^{\prime}$ be that part of $N$ which remains in the base locus for $|F|$ and let $N^{\prime \prime}$ be what remains in the base locus of $|F+L|$; thus $N^{\prime}=c_{1}^{\prime} C_{1}+\cdots+c_{r}^{\prime} C_{r}$ and $N^{\prime \prime}=c_{1}^{\prime \prime} C_{1}+\cdots+c_{r}^{\prime \prime} C_{r}$. Note that $c_{j}-c_{j}^{\prime} \leq \operatorname{deg}\left(C_{j}\right)$, because $-c_{j}=F_{t-1}(Z) \cdot C_{j}$ and $-c_{j} \leq-c_{j}^{\prime}=\min \left(F_{t}(Z) \cdot C_{j}, 0\right) \leq$ $L \cdot C_{j}+F_{t-1}(Z) \cdot C_{j}=\operatorname{deg}\left(C_{j}\right)-c_{j}$. By Lemma 2.1.1, dim cok $\mu_{t}=\operatorname{dim} \operatorname{cok} \mu_{F}=$ $\operatorname{dim} \operatorname{cok} \mu_{H+L+N-N^{\prime}}+\left(h^{0}(X, F+L)-h^{0}\left(X, H+2 L+N-N^{\prime}\right)\right)$. By Proposition 3.1 and Theorem 3.3 .

$$
\begin{aligned}
\operatorname{dim} \operatorname{cok} \mu_{H+L+N-N^{\prime}} & =\sum_{j} \operatorname{dim} \operatorname{cok} \mu_{L+\left(c_{j}-c_{j}^{\prime}\right) N_{j}} \\
& \leq \sum_{j}\left(\left(\begin{array}{c}
c_{j}-c_{j}^{\prime}-a_{C_{j}} \\
2
\end{array}\right)+\left(\begin{array}{c}
c_{j}-c_{j}^{\prime}-b_{C_{j}} \\
2
\end{array}\right)\right) .
\end{aligned}
$$

Since $N^{\prime \prime}$ is in the base locus of $|F+L|$, we have $h^{0}(X, F+L)=h^{0}\left(X, F+L-N^{\prime \prime}\right)$. But $F+L-N^{\prime \prime}=H+2 L+N-N^{\prime \prime}=H+2 L+N-N^{\prime}+\left(N^{\prime}-N^{\prime \prime}\right)$, and both $H+2 L+N-N^{\prime \prime}$ and $H+2 L+N-N^{\prime}$ are nef and effective, so by Conjecture 2.2.1 we have $h^{1}\left(X, H+2 L+N-N^{\prime}+\left(N^{\prime}-N^{\prime \prime}\right)\right)=0=$ $h^{1}\left(X, H+2 L+N-N^{\prime}\right)$. Plugging into Riemann-Roch and simplifying gives 
$h^{0}(X, F+L)-h^{0}\left(X, H+2 L+N-N^{\prime}\right)=h^{0}\left(X, H+2 L+N-N^{\prime}+\left(N^{\prime}-N^{\prime \prime}\right)\right)-$ $h^{0}\left(X, H+2 L+N-N^{\prime}\right)=\left(N^{\prime}-N^{\prime \prime}\right)^{2} / 2-K_{X} \cdot\left(N^{\prime}-N^{\prime \prime}\right) / 2+\left(2 L+H+N-N^{\prime}\right)$. $\left(N^{\prime}-N^{\prime \prime}\right)$. Keeping in mind that $\left(L+H+N-N^{\prime}\right) \cdot\left(N^{\prime}-N^{\prime \prime}\right)=0$, this gives $\sum_{j} d_{j}\left(c_{j}^{\prime}-c_{j}^{\prime \prime}\right)-\sum_{j}\left(c_{j}^{\prime}-c_{j}^{\prime \prime}\right)$. Putting everything together gives

$$
\begin{aligned}
\operatorname{dim} \operatorname{cok} \mu_{t} \leq & \sum_{j}\left(\left(\begin{array}{c}
c_{j}-c_{j}^{\prime}-a_{C_{j}} \\
2
\end{array}\right)+\left(\begin{array}{c}
c_{j}-c_{j}^{\prime}-b_{C_{j}} \\
2
\end{array}\right)\right) \\
& +\sum_{j} d_{j}\left(c_{j}^{\prime}-c_{j}^{\prime \prime}\right)-\sum_{j}\left(\begin{array}{c}
c_{j}^{\prime}-c_{j}^{\prime \prime} \\
2
\end{array}\right) .
\end{aligned}
$$

\section{ACKNOWLEDGMENTS}

The authors thank GNSAGA, and the University of Bologna, which supported visits to Bologna by the second author, who also thanks the NSA and NSF for supporting his research. We also thank the referee for his careful and helpful comments and J. Roé for a helpful comment.

\section{APPENDIX}

\section{A1. MAKing The SHGH CONJECture EXPLicit}

In this appendix we give another version of the SHGH Conjecture and show how to derive explicit predictions for values of Hilbert functions using it.

A1.1. The Weyl group. We now recall the Weyl group $W=W_{n}$, which acts on $\mathrm{Cl}(X)$ but which depends only on the number $n$ of points $P_{i}$ blown up. If $0 \leq n \leq 1$, then $W=\{i d\}$ is trivial. If $n=2$, then $W=\left\{i d, s_{1}\right\}$, where for any divisor class $F, s_{1}(F)=F+\left(r_{1} \cdot F\right) r_{1}$, where $r_{1}=E_{1}-E_{2}$. For $n>2$, let $r_{0}=L-E_{1}-E_{2}-E_{3}$ and for $1 \leq i<n$, let $r_{i}=E_{i}-E_{i+1}$. Then $W$ is generated by the operators $s_{i}$, $0 \leq i<n$, where $s_{i}(F)=F+\left(r_{i} \cdot F\right) r_{i}$. It is now easy to check that $W$ preserves the intersection form (i.e., $w F \cdot w G=F \cdot G$ for all $w \in W$ and all $F, G \in \mathrm{Cl}(X)$ ), and that $w K_{X}=K_{X}$ for all $w \in W$. The subgroup generated by $s_{1}, \ldots, s_{n-1}$ is just the permutation group on $E_{1}, \ldots, E_{n}$. The action of the element $s_{0}$ corresponds to that of the quadratic Cremona transformation centered at $P_{1}, P_{2}, P_{3}$.

If $n=0, X=\mathbf{P}^{2}$ has no exceptional curves. If $n=1$, then $E_{1}$ is the only exceptional curve, and if $n=2$, then $E_{1}, E_{2}$ and $L-E_{1}-E_{2}$ are the only exceptional curves. For $n \geq 3$, Nagata [N2 has shown that if a class $E$ is the class of an exceptional curve, then $E \in W E_{n}$; i.e., the classes of exceptional curves lie in a single $W$-orbit. Moreover, if $E \in W E_{n}$ and the points $P_{i}$ are general, Nagata showed $E$ is the class of an exceptional curve. (When $E \in W E_{n}$ but the points are not general, then although $E$ is effective, it can fail to be reduced and irreducible, and thus need not be the class of an exceptional curve. For example, $2 L-E_{1}-$ $\cdots-E_{5} \in W E_{5}$, but if $P_{1}, P_{2}, P_{3}$ are collinear, then the proper transform of the line through $P_{1}, P_{2}, P_{3}$ is a fixed component of $\left.\left|2 L-E_{1}-\cdots-E_{5}\right|.\right)$

If $n=0$, let $\mathcal{E}_{n}$ be the submonoid of $\operatorname{Cl}(X)$ generated by $L$. If $n=1$, let $\mathcal{E}_{n}$ be the submonoid generated by $L-E_{1}$ and $E_{1}$. If $n=2$, let $\mathcal{E}_{n}$ be generated by $L-E_{1}-E_{2}, E_{1}$ and $E_{2}$, while if $n \geq 3$, let $\mathcal{E}_{n}$ be generated by all exceptional curves (or equivalently by the orbit $W E_{n}$ of $E_{n}$ under $W$ ). Thus if $n \geq 3$, every element $D \in \mathcal{E}_{n}$ is of the form $D=\sum c_{i} C_{i}$, where $c_{i}$ is a nonnegative integer and 
$C_{i}$ is an exceptional curve. Define $\mathcal{E}_{n}^{*}$ to be the dual cone; thus $F \in \mathcal{E}_{n}^{*}$ means that $F \cdot D \geq 0$ for every $D \in \mathcal{E}_{n}$ (and thus that $F \cdot E \geq 0$ for every exceptional curve $E)$.

Let $\mathrm{EFF}=\operatorname{EFF}(X) \subset \mathrm{Cl}(X)$ denote the submonoid of classes of effective divisors, let $\mathrm{NEF}=\mathrm{NEF}(X) \subset \mathrm{Cl}(X)$ denote the submonoid (indeed the cone, since a class is nef if a positive multiple is) of classes of nef divisors, let $\Psi_{n}$ be the submonoid generated by the union of $\mathcal{E}_{n}$ and the element $-K_{X}$ and let $\Delta_{n}$ be the submonoid of $\mathrm{Cl}(X)$ generated by $H_{0}=L, H_{1}=L-E_{1}, H_{2}=2 L-E_{1}-E_{2}$, and $H_{i}=-K_{X}+E_{i+1}+\cdots+E_{n}=3 L-E_{1}-\cdots-E_{i}$, for $3 \leq i \leq n$. Notice that $F=t L-m_{1} E_{1}-\cdots-m_{n} E_{n} \in \Delta_{n}$ if and only if $t \geq m_{1}+m_{2}+m_{3}$ and $m_{1} \geq m_{2} \geq \cdots \geq m_{n} \geq 0$, and that $H_{n}$ is $-K_{X}$ and $H_{i} \cdot H_{j} \geq 0$ unless $i, j \geq 10$.

Since the next result is a statement for all classes on $X$, we need to state it in terms of a blowing up of generic points. However, when we are interested in a specific class $F$, it is enough to consider a blowup of general points (but the conditions of generality will depend on $F$ ). The following result is known but hard to cite.

Lemma A1.1.1. Let $X$ be the blowup of $\mathbf{P}^{2}$ at $n$ generic points $P_{i}$.

(a) If $A \in \Delta_{n}$ and $w \in W_{n}(X)$, then $w A=A+a_{0} r_{0}+\cdots+a_{n-1} r_{n-1}$ for some nonnegative integers $a_{i}$.

(b) $\operatorname{NEF}(X) \subset \mathcal{E}_{n}^{*}=W_{n} \Delta_{n} \subset \Psi_{n}$.

(c) $h^{j}(X, F)=h^{j}(X, w F)$ for all $j$, all $w \in W_{n}$ and all $F \in C l(X)$.

(d) $\operatorname{EFF}(X) \subset \Psi_{n}$.

(e) If $F \in \Psi_{n}$, then there is a unique decomposition $F=H+N$ where $H \in \mathcal{E}_{n}^{*}$, $N \in \mathcal{E}_{n}, H \cdot N=0$ and, if $N \neq 0$, then $N=c_{1} C_{1}+\cdots+c_{r} C_{r}$ where for each $i, c_{i}$ is a positive integer and $C_{i}$ is the class of an exceptional curve with $C_{i} \cdot C_{j}=0$ for all $i \neq j$. Moreover, $H$ is effective if $F$ is.

Proof. (a) See, for example, Lemma 1.2 (1) of [Ha5]. (b) First note that NEF $\subset \mathcal{E}_{n}^{*}$, since $\mathcal{E}_{n} \subset \mathrm{EFF}$. Now we verify that $\mathcal{E}_{n}^{*}=W_{n} \Delta_{n}$. We leave the cases $0 \leq n<3$ to the reader; assume $n \geq 3$. It is known that $\mathrm{EFF}$ and $\mathrm{NEF}$ are $W_{n}$-invariant and that the set of exceptional divisors is just the orbit $W_{n} E_{n}$; cf. [N2]. Since $W_{n}$ preserves the set of exceptional curves, if we show $\Delta_{n} \subset \mathcal{E}_{n}^{*}$, then $W_{n} \Delta_{n} \subset \mathcal{E}_{n}^{*}$. But $H_{i}$ is nef (hence in $\mathcal{E}_{n}^{*}$ ) for $i \leq 9$. For $i>9, H_{i}=-K_{X}+E_{i+1}+\cdots+E_{n}$; hence for any exceptional curve $E$ we have $H_{i} \cdot E \geq 0$, since $E$ meets $-K_{X}$ once and $E \cdot E_{j} \geq-1$ with equality if and only if $E=E_{j}$. Thus $\Delta_{n} \subset \mathcal{E}_{n}^{*}$.

Conversely, say $F \in \mathcal{E}_{n}^{*}$. Note that every element $D \in \mathcal{E}_{n}^{*}$ satisfies $D \cdot L \geq 0$, since $L=\left(L-E_{1}-E_{2}\right)+E_{1}+E_{2}$. Since $W_{n}$ preserves $\mathcal{E}_{n}^{*}$, there must be some $w \in W_{n}$ such that $L \cdot w F$ is as small as possible. Thus, $w F \cdot r_{0} \geq 0$; otherwise we would have $s_{0} w F \cdot L<w F \cdot L$. We can also assume $m_{1} \geq m_{2} \geq \cdots \geq m_{n}$, where $m_{i}=w F \cdot E_{i}$, since each operator $s_{i}, i>0$, merely transposes $E_{i}$ and $E_{i+1}$, so we can in $W_{n}$ permute the $E_{i}$ without affecting $L \cdot w F$. Thus $w F \cdot r_{i} \geq 0$ for all $i \geq 1$. Finally, $w F \cdot\left(L-E_{1}-E_{2}\right) \geq 0$ since $w F \in \mathcal{E}_{n}^{*}$, and $w F \cdot\left(L-E_{1}\right) \geq 0$ since $L-E_{1}=\left(L-E_{1}-E_{2}\right)+E_{2}$ is a sum of exceptional curves. By Lemma 1.4 of A.H2, we thus have $w F \in \Delta_{n}$.

Finally, it is clear that $\Delta_{n} \subset \Psi_{n}$ but $\Psi_{n}$ is $W_{n}$-invariant, so $W_{n} \Delta_{n} \subset \Psi_{n}$.

(c) This is, in somewhat different language, due to Nagata N2]. The basic idea is this. Say $F=t L-m_{1} E_{1}-\cdots-m_{n} E_{n}$. Then $w F=t w L-m_{1} w E_{1}-\cdots-m_{n} w E_{n}$, where $L^{\prime}=w L, E_{1}^{\prime}=w E_{1}, \ldots, E_{n}^{\prime}=w E_{n}$ is a basis of $\mathrm{Cl}(X)$. This basis is, however, an exceptional configuration; i.e., there is a birational morphism $p^{\prime}: X \rightarrow$ 
$\mathbf{P}^{2}$ such that $E_{i}^{\prime}=p^{-1}\left(P_{i}^{\prime}\right)$ for some points $P_{i}^{\prime} \in \mathbf{P}^{2}$ and such that $L^{\prime}$ is the pullback via $p^{\prime}$ of the class of a line in $\mathbf{P}^{2}$ (see Theorem 0.1 of [A.H1]), but the points $P_{i}^{\prime}$ are themselves generic. Since $P_{i}$ and $P_{i}^{\prime}$ both give generic sets of points, all that matters are the coefficients $t$ and $m_{i}$, so we have $h^{j}\left(X, t L-m_{1} E_{1}-\cdots-m_{n} E_{n}\right)=$ $h^{j}\left(X, t L^{\prime}-m_{1} E_{1}^{\prime}-\cdots-m_{n} E_{n}^{\prime}\right)$; i.e., $h^{j}(X, F)=h^{j}(X, w F)$.

(d) Let $F \in \operatorname{EFF}(X)$. Then there are at most finitely many exceptional curves $E$ such that $F \cdot E<0$. Let this finite set of distinct exceptional curves be $C_{1}, \ldots, C_{r}$, let $c_{i}=-F \cdot C_{i}$, let $N=c_{1} C_{1}+\cdots+c_{r} C_{r}$ and let $H=F-N$. Note that $C_{i} \cdot C_{j}=0$ for all $i \neq j$ (since $\left(C_{i}+C_{j}\right) \cdot F<0$, but $C_{i} \cdot C_{j}>0$ implies $\left(C_{i}+C_{j}\right)$ meets both $C_{i}$ and $C_{j}$ nonnegatively and hence is nef) and that $H \cdot C_{i}=0$. Since $F$ is effective, $N$ is contained in the scheme theoretic base locus of $|F|$; hence $H$ is effective. But if $H \cdot E<0$, then $E$ is not $C_{i}$ for any $i$; hence $E \cdot F \geq 0$, so we get $E \cdot C_{i}>0$ (implying $E+C_{i}$ is nef) for some $i$ even though $H \cdot\left(E+C_{i}\right)<0$. It follows that $H \in \mathcal{E}_{n}^{*}$. The result follows since $N \in \mathcal{E}_{n} \subset \Psi_{n}$ and $H \in \mathcal{E}_{n}^{*} \subset \Psi_{n}$.

(e) We leave the cases $0 \leq n<3$ to the reader, so assume $n \geq 3$. If $F \in \Psi_{n}$, then $w F \cdot L \geq 0$ and $w F \cdot\left(L-E_{1}\right) \geq 0$ for all $w \in W_{n}$, since $w F \in \Psi_{n}$ but $L$ and $L-E_{1}$ are nef and meet $-K_{X}$ nonnegatively. Choose $w$ such that $w F \cdot L$ is as small as possible, and write $w F=t L-m_{1} E_{1}-\cdots-m_{n} E_{n}$. Since $W_{n}$ includes the group of permutations of $E_{1}, \ldots, E_{n}$, we may assume that $m_{1} \geq \cdots \geq m_{n}$. Since $t$ is as small as possible, we know that $t \geq m_{1}+m_{2}+m_{3}$ (otherwise $s_{0} w F \cdot L<w F \cdot L$ ). If $m_{3} \geq 0$ or $m_{2} \leq 0$, let $H^{\prime}=t L-\sum_{m_{i}>0} m_{i} E_{i}$ and $N^{\prime}=-\sum_{m_{i}<0} m_{i} E_{i}$. Using the definition of $\Delta_{n}$ and the facts that $t \geq m_{1}+m_{2}+m_{3}$ and $t \geq m_{1}$, it is now not hard to check that $H^{\prime} \in \Delta_{n}$, and clearly $N^{\prime} \in \mathcal{E}_{n}$. If $m_{3}<0$ and $m_{2}>0$, there are two cases. If $c=\left(t L-m_{1} E_{1}-m_{2} E_{2}\right) \cdot\left(L-E_{1}-E_{2}\right)<0$, then let $H^{\prime}=(t+c) L-\left(m_{1}+c\right) E_{1}-\left(m_{2}+c\right) E_{2}$, and let $N^{\prime}=(-c)\left(L-E_{1}-E_{2}\right)-m_{3} E_{3}-$ $\cdots-m_{n} E_{n}$. If $\left(t L-m_{1} E_{1}-m_{2} E_{2}\right) \cdot\left(L-E_{1}-E_{2}\right) \geq 0$, let $H^{\prime}=t L-m_{1} E_{1}-m_{2} E_{2}$, and let $N^{\prime}=-m_{3} E_{3}-\cdots-m_{n} E_{n}$. Either way $H^{\prime} \in \Delta_{n}$ and $N^{\prime} \in \mathcal{E}_{n}$. In all cases we also have $H^{\prime} \cdot N^{\prime}=0$ and that the components of $N^{\prime}$ are disjoint and orthogonal to $H^{\prime}$. We now take $H=w^{-1} H^{\prime}$ and $N=w^{-1} N^{\prime}$, where the classes $C_{i}$ are the $w^{-1}$ translates of the components of $N^{\prime}$. Uniqueness follows from the fact that $N=\sum_{E \text { exceptional }}-(F \cdot E) E$. Note that in case $F$ is effective we found a decomposition $F=H+N$ in (d), with $H$ effective. Uniqueness now shows that $H$ is necessarily effective.

Remark A1.1.2. As an application of Lemma A1.1.1, we will classify all smooth rational curves $C$ on a blowup $p: X \rightarrow \mathbf{P}^{2}$ of general points, either by assuming the SHGH Conjecture, or by working over the complex numbers using Proposition 2.4 of $\mathrm{dF}$, assuming that the points blown up are very general. In either case, we have $C^{2} \geq-1$. If $C^{2}=-1$, then $C$ is an exceptional curve. If $C^{2}>-1$, then $C$ is nef; hence $w C \in \Delta$ for some $w \in W$ by LemmaA1.1.1(b), so it suffices if we find all smooth rational $C \in \Delta$. Since $C \in \Delta$, we have $C=\sum_{i} a_{i} H_{i}$ for some nonnegative integers $a_{i}$. Note that $C \cdot H_{j} \geq 0$ for $0 \leq j \leq 2$. By adjunction and $C^{2}>-1$ we have $-C \cdot K_{X}=C^{2}+2 \geq 2$. Since $C$ is nef, we have $C \cdot\left(E_{j+1}+\cdots+E_{n}\right) \geq 0$. Thus $C \cdot H_{j}=C \cdot\left(-K_{X}+E_{j+1}+\cdots+E_{n}\right) \geq 2$ for all $j>2$. If $a_{j}>0$ for some $j>2$, let $C^{\prime}=C-H_{j}$. Since $C^{\prime}$ is still a nonnegative integer combination of the $H_{i}$, we have $C \cdot C^{\prime} \geq 0$. Now $C^{2}=C \cdot C^{\prime}+C \cdot H_{j} \geq C \cdot H_{j} \geq C \cdot H_{n}=-C \cdot K_{X}=C^{2}+2$. I.e., we must have $a_{j}=0$ for all $j>2$.

Thus $C=a H_{0}+b H_{1}+c H_{2}$. It is now an easy exercise using adjunction to show that the only solutions to $C^{2}+C \cdot K_{X}=-2$ are $H_{0}, 2 H_{0}, H_{1}, H_{2}, H_{0}+b H_{1}$ and 
$H_{2}+b H_{1}$. Thus the $W$ orbits of these and $E_{1}$ are the only possible smooth rational curves in $X$.

Each such $C$ can be turned into an exceptional curve $E$ by subtracting off additional $E_{i}$; for example, if $C=H_{2}+2 H_{1}=4 L-3 E_{1}-E_{2}$, then $E=$ $4 L-3 E_{1}-E_{2}-\cdots-E_{9}$ is an exceptional curve. Moreover, $\left.p^{*} \Omega(1)\right|_{C}$ has the same splitting as does $\left.p^{*} \Omega(1)\right|_{E}$, since $C$ and $E$ both have the same image $p(C)=p(E)$ in $\mathbf{P}^{2}$.

Thus an algorithm for computing the splitting for exceptional curves handles all smooth rational $C$.

A1.2. The SHGH conjecture. Given any class $F \in \mathrm{Cl}(X)$, there is a geometrically defined quantity $e\left(h^{0}, F\right)$ such that $h^{0}(X, F) \geq e\left(h^{0}, F\right)$ holds for general points $P_{i}$. We now define this lower bound.

If $F \notin \Psi_{n}$, then $h^{0}(X, F)=0$ by Lemma A1.1.1, and we set $e\left(h^{0}, F\right)=0$. If $F \in \Psi_{n}$, then we have the decomposition $F=H+N$ given by Lemma A1.1.1(e), and we have $h^{0}(X, F)=h^{0}(X, H)$. Since $H \cdot L \geq 0$, we have $h^{0}(X, H) \geq$ $\max \left(0,\left(H^{2}-K_{X} \cdot H\right) / 2+1\right)$ and we set $e\left(h^{0}, F\right)=\max \left(0,\left(H^{2}-K_{X} \cdot H\right) / 2+1\right)$. Clearly, $h^{0}(X, F) \geq e\left(h^{0}, F\right)$ holds. We can now state the SHGH Conjecture, which says that equality in fact holds:

Conjecture A1.2.1. We have $h^{0}(X, F)=e\left(h^{0}, F\right)$, where $F \in C l(X)$ and $X$ is the blowup of $\mathbf{P}^{2}$ at general points $P_{i}$.

A version of the SHGH Conjecture for fat points follows from this. Given a fat point subscheme $Z=m_{1} P_{1}+\cdots+m_{n} P_{n}$ supported at general points $P_{i}$, we define $e\left(h_{Z}, t\right)$ to be $e\left(h^{0}, F_{t}(Z)\right)$, where $F_{t}(Z)=t L-m_{1} E_{1}-\cdots-m_{n} E_{n}$.

Conjecture A1.2.2. We have $h_{Z}(t)=e\left(h_{Z}, t\right)$, where $Z=m_{1} P_{1}+\cdots+m_{n} P_{n}$ is a fat point scheme supported at general points $P_{i}$ of $\mathbf{P}^{2}$.

To apply these conjectures, one must be able to compute $e\left(h^{0}, F\right)$. We give two examples showing how to do so.

Example A1.2.3. Suppose $F=t L-\left(77\left(E_{1}+\cdots+E_{7}\right)+44 E_{8}+11 E_{9}+11 E_{10}+\right.$ $\left.11 E_{11}\right)$. To compute $e\left(h^{0}, F\right)$ for any given $t$, just mimic the proof of Lemma A1.1.1(e). The idea is to find an element $w \in W$ such that either $w F \cdot L$ is as small as possible, or $w F \cdot L<0$ or $w F \cdot\left(L-E_{1}\right)<0$. For example, say $t=208$. Apply $s_{0}$ to $F$ to get $s_{0} F=185 L-54 E_{1}-54 E_{2}-54 E_{3}-77 E_{4}-77 E_{5}-77 E_{6}-77 E_{7}-44 E_{8}-$ $11 E_{9}-11 E_{11}-11 E_{11}$. Permute the $E_{i}$ so that the coefficients are nondecreasing, which gives $F^{\prime}=185 L-77 E_{1}-77 E_{2}-77 E_{3}-77 E_{4}-54 E_{5}-54 E_{6}-54 E_{7}-44 E_{8}-$ $11 E_{9}-11 E_{11}-11 E_{11}$. This operation, taking $F$ to $F^{\prime}$, is now repeated until we obtain a class $F^{\prime \prime}$ such that either $F^{\prime \prime} \cdot L<0$, or $F^{\prime \prime} \cdot\left(L-E_{1}\right)<0$, or until $F^{\prime \prime} \cdot L \geq 0$, $F^{\prime \prime} \cdot\left(L-E_{1}\right) \geq 0$ and $F^{\prime \prime} \cdot r_{0} \geq 0$. In this case the class $F^{\prime \prime}$ we eventually end up with is $-23 L-8 E_{1}+E_{2}+5 E_{3}+5 E_{4}+5 E_{5}+5 E_{6}+8 E_{7}+8 E_{8}+14 E_{9}+17 E_{10}+17 E_{11}$; hence $e\left(h^{0}, F\right)=0$, since $F^{\prime \prime} \cdot L<0$ (so $F^{\prime \prime} \notin \Psi_{11}$ ). If, for example, $t=209$, then the class we end up with is $F^{\prime \prime}=11 E_{11}$, so the decomposition of Lemma A1.1.1(d) is $H=0$ and $N=F$, so $e\left(h^{0}, F\right)=1$. If $t=210$, then we end up with $F^{\prime \prime}=27 L-8\left(E_{1}+\cdots+E_{4}\right)-5\left(E_{5}+\cdots+E_{11}\right)$, which is in $\Delta$, so $F=H, N=0$, and $e\left(h^{0}, F\right)=\left(H^{2}-K_{X} \cdot H\right) / 2+1$. In this example, the SHGH Conjecture, that $h^{0}(X, F)=e\left(h^{0}, F\right)$, in fact holds for all $t$. It holds for $t<209$ since $F \notin \Psi_{n}$ for those $t$. It holds for $t=209$, since $w F=11 E_{11}$ for some $w$, so $h^{0}(X, F)=h^{0}\left(X, 11 E_{11}\right)=1$. Also, it holds for $t>209$ since for these cases, 
$F \in \mathcal{E}_{11}^{*}=W_{11} \Delta_{11}$, so $F=H$, and $-K_{X} \cdot F \geq 0$, so $h^{0}(X, F)=\left(F^{2}-K_{X} \cdot F\right) / 2+1$ by Theorem 1.1 of [A.H2 and semicontinuity of $h^{0}$. (Macaulay 2 scripts for carrying out both the LemmaA1.1.1(d) decomposition and the Weyl group calculations, and a sample Macaulay 2 session demonstrating their use, are included in Section A2.3 of the posted version of this paper, GHI2.)

Example A1.2.4. Now consider $F=t L-50 E_{1}-50 E_{2}-38 E_{3}-38 E_{4}-26 E_{5}-$ $26 E_{6}-22 E_{7}-18 E_{8}-14 E_{9}-14 E_{10}$. As in Example A1.2.3, we have $e\left(h^{0}, F\right)=0$ for $t<102$, since $F \notin \Psi_{10}$. For $t=102$, we find a $w$ such that $w F=6 L-$ $2\left(E_{2}+\cdots+E_{8}\right)+2 E_{9}+6 E_{10}$. Thus the decomposition $F=H+N$ has $H=$ $w^{-1}\left(6 L-2\left(E_{2}+\cdots+E_{8}\right)\right)$ and $N=w^{-1}\left(2 E_{9}+6 E_{10}\right)$, where $w^{-1}$ can be performed by simply reversing the operations which gave $w$. What we find is $H=38 L-18 E_{1}-$ $18 E_{2}-14 E_{3}-14 E_{4}-10 E_{5}-10 E_{6}-8 E_{7}-8 E_{8}-6 E_{9}-6 E_{10}$, and $N=2 C_{1}+6 C_{2}$, where $C_{1}=8 L-4 E_{1}-4 E_{2}-3 E_{3}-3 E_{4}-2 E_{5}-2 E_{6}-E_{7}-2 E_{8}-E_{9}-E_{10}$ and $C_{2}=8 L-4 E_{1}-4 E_{2}-3 E_{3}-3 E_{4}-2 E_{5}-2 E_{6}-2 E_{7}-E_{8}-E_{9}-E_{10}$. Thus $h^{0}(X, F)=h^{0}(X, H)$, and it is known that $h^{0}(X, H)=4$. For $t \geq 103$, we have $F=H$ and $N=0$. In fact, for $t=103$ we have $h^{0}(X, F)=92$, and for $t=104$ we have $h^{0}(X, F)=197$. (For the same reasons as in Example A1.2.3, the SHGH Conjecture holds for $F$ for all $t$.)

Given a fat point subscheme $Z \subset \mathbf{P}^{2}$ with general support, we can now define the expected value $e(g .(Z), i)$ of the Betti number $g_{i}(Z)$ for $i>\alpha+1$ :

Definition A1.2.5. Let $F=F_{i-2}(Z)$; note that $F$ is effective, since $i>\alpha+1$. Thus we have a decomposition $F=H+N$, with $N=c_{1} C_{1}+\cdots+c_{r} C_{r}$, as in Lemma A1.1.1(e). Let $m_{i}=\min \left(c_{i}, L \cdot C_{i}\right)$. Let $M=m_{1} C_{1}+\cdots+m_{r} C_{r}$, so $N-M$ is effective and $F=H+M+(N-M)$. Then $\operatorname{dim} \operatorname{cok} \mu_{L+H+M}=\operatorname{dim} \operatorname{cok} \mu_{L+M}$ by Proposition 3.1 assuming the SHGH Conjecture, so $g_{i}(Z)=\operatorname{dim} \operatorname{cok} \mu_{L+F}=$ $\operatorname{dim} \operatorname{cok} \mu_{L+M}+\left(h^{0}(X, 2 L+F)-h^{0}(X, 2 L+H+M)\right)$ by Lemma 2.1.1, and $\operatorname{dim} \operatorname{cok} \mu_{L+M} \leq \sum_{i}\left(\begin{array}{c}m_{i}-b_{C_{i}} \\ 2\end{array}\right)+\left(\begin{array}{c}m_{i}-a_{C_{i}} \\ 2\end{array}\right)$ by Theorem 3.3 with equality assuming Conjecture 3.4. Thus we take $e(g .(Z), i)$ to be

$$
\left(h^{0}(X, 2 L+F)-h^{0}(X, 2 L+H+M)\right)+\sum_{i}\left(\begin{array}{c}
m_{i}-b_{C_{i}} \\
2
\end{array}\right)+\left(\begin{array}{c}
m_{i}-a_{C_{i}} \\
2
\end{array}\right) .
$$

(In the notation of the proof of Theorem 1.2.1, $M$ is $N-N^{\prime}$ and $m_{i}=c_{i}-c_{i}^{\prime}$, so the upper bound in Theorem 1.2.1 is, by the proof of Theorem 1.2.1, exactly $e(g .(Z), i)$.

The following result will be useful in our examples. Versions of this result were proved in [Ha2] and [FHH] and were the basis for the results in [Ha1] and [FHH].

Lemma A1.2.6. Let $Z=m_{1} P_{1}+\cdots+m_{n} P_{n}$ be a fat point subscheme of $\mathbf{P}^{2}$. Assume $F=F_{k}(Z)$ is the class of an effective divisor on $X$, and define $h(F)=$ $h^{0}(X, F), l(F)=h^{0}\left(X, F-\left(L-E_{1}\right)\right), l^{*}(F)=h^{1}\left(X, F-\left(L-E_{1}\right)\right), q(F)=$ $h^{0}\left(X, F-E_{1}\right)$, and $q^{*}(F)=h^{1}\left(X, F-E_{1}\right)$. Then

$$
l(F) \leq \operatorname{dim} \operatorname{ker} \mu_{F} \leq l(F)+q(F) .
$$

If moreover $h^{1}(X, F)=0$, then

$$
k+2-2 h(F)+l(F) \leq \operatorname{dim} \operatorname{cok} \mu_{F} \leq q^{*}(F)+l^{*}(F) .
$$

Here are two examples showing explicitly how to compute expected values of the graded Betti numbers. 
Example A1.2.7. Suppose we want to determine the graded Betti numbers of the fat points subscheme $Z=m_{1} P_{1}+\cdots+m_{n} P_{n} \in \mathbf{P}^{2}$, where $n=10$ here, the points $P_{i}$ are general and the sequence of multiplicities $m_{i}$ is $(50,50,38,38,26,26,22,18$, 14, 14). We found the Hilbert function in Example A1.2.4, from which it follows that $g_{i}=0$ except for $i=102$ (where we have $g_{102}=h_{Z}(102)=4$ ) and possibly for $i=$ 103 and $i=104$. We do not have an expected value for $g_{103}$, since $103=\alpha(Z)+1$, but an ad hoc use of Lemma A1.2.6 gives dim ker $\mu_{F_{102}(Z)} \leq l\left(F_{102}(Z)\right)+q\left(F_{102}(Z)\right)$, where $l\left(F_{102}(Z)\right)=h^{0}\left(X, F_{102}(Z)-\left(L-E_{1}\right)\right)$ and $q\left(F_{102}(Z)\right)=h^{0}\left(X, F_{102}(Z)-E_{1}\right)$. Neither $F_{102}(Z)-\left(L-E_{1}\right)$ nor $F_{102}(Z)-E_{1}$ is in $\Psi$, so $l=q=0$, so $\mu_{F_{102}(Z)}$ is injective; hence $g_{103}=\operatorname{dim} \operatorname{cok} \mu_{F_{102}(Z)}=h^{0}\left(X, F_{103}(Z)\right)-3 h^{0}\left(X, F_{102}(Z)\right)=$ $92-3(4)=80$. To compute $g_{104}$, recall in Example A1.2.4 we found $F_{103}(Z)=$ $H+2 C_{1}+6 C_{2}$. From Lemma 2.1.2 we have $a_{C_{i}}=b_{C_{i}}=4$ for $i=1,2$. Since $h^{1}(X, H)=0$ holds here, by Proposition 3.1 we have $g_{104}=\operatorname{dim} \operatorname{cok} \mu_{F_{103}(Z)}=$ $\operatorname{dim} \operatorname{cok} \mu_{L+2 C_{1}}+\operatorname{dim} \operatorname{cok} \mu_{L+6 C_{2}}$. By Theorem 3.3 we have $\operatorname{dim} \operatorname{cok} \mu_{L+2 C_{1}}=0$ and $\operatorname{dim} \operatorname{cok} \mu_{L+6 C_{2}}=2$, so $g_{104}=2$. For $i \geq 104$, we have $h^{1}\left(X, F_{i}(Z)\right)=0$; hence $g_{i+1}=0$ by Corollary 2.3.2

We can now write down a minimal free graded resolution for $I(Z)$. It is $0 \rightarrow$ $M_{1} \rightarrow M_{0} \rightarrow I(Z) \rightarrow 0$, where $M_{0}=R^{2}[-104] \oplus R^{80}[-103] \oplus R^{4}[-102]$, and from the Hilbert functions of $I(Z)$ and $M_{0}$ we now find $M_{1}=R^{16}[-105] \oplus R^{69}[-104]$.

Example A1.2.8. Consider $Z=48 P_{1}+33 P_{2}+33 P_{3}+33 P_{4}+32 P_{5}+32 P_{6}+$ $32 P_{7}+24 P_{8}+16 P_{9}$, where the points $P_{i}$ are general. Then $h_{Z}(t)=0$ for $t<98$, since $F_{t}(Z) \notin \Psi$, and $h_{Z}(t)=\left(\begin{array}{c}t+2 \\ 2\end{array}\right)-4879$ for $t \geq 98$, since then $h_{Z}(t)>0$, $F_{t}(Z) \in \mathcal{E}^{*}$ and the SHGH Conjecture is known to hold for $n=9$ general points (see [N2], or use the fact that any nef divisor $F$ on a blowup of $\mathbf{P}^{2}$ at 9 general points has $-K_{X} \cdot F \geq 0$ and apply the results of [A.H2]). We also know that $g_{t}=0$ for $t<98$, and $g_{98}=h_{Z}(98)=71$. By Corollary 2.3.2. we have $g_{t}=0$ for $t>99$. In this case $\alpha=98$, and we do not in general have a conjectural value for $g_{\alpha+1}$, but in this case there is an element $w \in W$ such that $F_{97}(Z)=L+H+N$, where $H=L-E_{2}-E_{3}-E_{4}, N=8 E$, and where $E$ is the exceptional curve $12 L-6 E_{1}-4 E_{2}-\cdots-4 E_{7}-3 E_{8}-2 E_{9}$. Since $h^{1}(X, H)=0$, reasoning as in the proof of Proposition 3.1, the cokernels of $\mu_{L+H+8 E}$ and $\mu_{L+8 E}$ are both isomorphic to the cokernel of $\mu_{E ; L+8 E}$ and hence to each other. By Lemma 2.1.2 we have $a_{e}=6=b_{E}$, so by Theorem 3.3 we have $g_{99}=\operatorname{dim} \operatorname{cok} \mu_{L+8 E}=2$. The minimal free graded resolution for $I(Z)$ is thus $0 \rightarrow R^{28}[-100] \oplus R^{44}[-99] \rightarrow$ $R^{2}[-99] \oplus R^{71}[-98] \rightarrow I(Z) \rightarrow 0$.

\section{A2. Computational aspects}

In this section we discuss various computational aspects of the problem of computing graded Betti numbers, partly to explain how our geometric approach can be used to make computer calculations more efficient, and partly to give additional evidence in support of Conjecture 3.4 .

A2.1. Splitting types. The first issue is the need to determine the splitting of the restriction of $p^{*} \Omega_{\mathbf{P}^{2}}$ (1) to a smooth rational curve $C \subset X$, where $p: X \rightarrow \mathbf{P}^{2}$ is a blowup of general points. We are mainly interested in doing this for exceptional curves, but it is of interest also to consider any smooth rational curve $C$. However, doing so for exceptional curves suffices to do it for all other smooth rational $C$ (see Remark A1.1.2). 
So suppose $E$ is an exceptional curve on $X$. The simplest approach conceptually is to find a Cremona transformation $w$ of the plane that transforms $p(E)$ to a line $A$. Pick a general basis $f_{1}, f_{2}, f_{3}$ of the linear forms on $\mathbf{P}^{2}$ and find their images $g_{i}=f_{i} \circ w^{-1}$ under $w$. Given the equation of $A$, which is easy to get since $\operatorname{deg}(A)=1$, we can find the ideal $J^{\prime}$ generated by the restrictions of the $g_{i}$ to $A$. This ideal typically has base points; the ideal $J$ residual to the base points is just the ideal generated by the restriction of the $f_{i}$ to $E$, but regarding $A=E$ as $\mathbf{P}^{1}$, we can find a minimal free resolution of the ideal over $K\left[\mathbf{P}^{1}\right]$. The degree of the syzygy of least degree is $a_{E}$; then $b_{E}=d-a_{E}$, where $d$ is the degree of $p(E)$.

It is not hard to convert this conceptual algorithm into code. For speed, all of the actual symbolic operations should be done in $K\left[\mathbf{P}^{1}\right]$ rather than in $K\left[\mathbf{P}^{2}\right]$. That is, one does not actually want to find the $g_{i}$ first and then restrict to the line. A discussion for how to push all of the computations down to $K\left[\mathbf{P}^{1}\right]$ is included in Section A2 of the posted version of the paper, GHI2. In addition, an explicit script that implements the computation and which is very fast is included in Section A2.3 of the posted version.

It would be nice to be able to predict what $a_{E}$ and $b_{E}$ should be, based only on knowing $t$ and the $m_{i}$, given a class $[E]=t L-m_{1} E_{1}-\cdots-m_{n} E_{n}$. This seems to be a difficult problem, with Lemma 2.1.2 being the main result. However, computational data suggests a possible new constraint on the splitting type for a smooth rational curve $C$ with $C^{2}=1$. Using sequences analogous to ( $\left.\ddagger\right)$ we get homomorphisms

$$
0 \rightarrow \operatorname{cok} \mu_{1 C} \rightarrow \operatorname{cok} \mu_{2 C} \rightarrow \operatorname{cok} \mu_{3 C} \rightarrow \cdots .
$$

The cokernel of cok $\mu_{i C} \rightarrow \operatorname{cok} \mu_{(i+1) C}$ is

$$
\operatorname{cok} \mu_{C ;(i+1) C} \cong H^{1}\left(C, \mathcal{O}_{C}\left(i+1-a_{C}\right) \oplus \mathcal{O}_{C}\left(i+1-b_{C}\right)\right) .
$$

For $r \geq b_{C}-2$, we thus get an upper bound $\operatorname{dim} \operatorname{cok} \mu_{r C} \leq(a-1)(a-2) / 2+$ $(b-1)(b-2) / 2$ by adding up the dimensions of the cokernels of cok $\mu_{C ;(i+1) C}$ for $i \leq r$.

Moreover, $C=w L$ for some $w \in W(X)$; hence $C$ is part of an exceptional configuration; i.e., $C=w L, C_{1}=w E_{1}, \ldots, C_{n}=w E_{n}$. When $r$ is big enough, $|r C-L|$ is nonempty and the fixed part of $|r C-L|$ is $d_{1} C_{1}+\cdots+d_{n} C_{n}$, where $d_{i}=C_{i} \cdot L$. Thus, by Proposition 3.1, Conjecture 3.4 and the SHGH Conjecture, we have $\operatorname{dim} \operatorname{cok} \mu_{r C}=\left(\left(a_{1}^{2}-a_{1}\right) / 2+\left(b_{1}^{2}-b_{1}\right) / 2\right)+\cdots+\left(\left(a_{n}^{2}-a_{n}\right) / 2+\left(b_{n}^{2}-b_{n}\right) / 2\right)$, where $\left(a_{i}, b_{i}\right)$ is the splitting type for $C_{i}$. This gives the inequality

$$
\begin{aligned}
\left(\left(a_{1}^{2}-a_{1}\right) / 2+\left(b_{1}^{2}-b_{1}\right) / 2\right)+\cdots+ & \left(\left(a_{n}^{2}-a_{n}\right) / 2+\left(b_{n}^{2}-b_{n}\right) / 2\right) \\
& \leq(a-1)(a-2) / 2+(b-1)(b-2) / 2 .
\end{aligned}
$$

This can be enough to determine $\left(a_{C}, b_{C}\right)$. For example, let $C=12 L-5 E_{1}-$ $5 E_{2}-5 E_{3}-4 E_{4}-4 E_{5}-4 E_{6}-4 E_{7}-2 E_{8}$. So C comes from a plane curve of degree 12 with three points of multiplicity 5 , four of multiplicity 4 and one of multiplicity 2. By Lemma 2.1.2, $\left(a_{C}, b_{C}\right)$ is either $(6,6)$ or $(5,7)$. Here are the $C_{i}$ and their types $\left(a_{i}, b_{i}\right)$. For convenience we only give the coefficients of $L,-E_{1}, \ldots,-E_{8}$ followed by the splitting type:

$$
\begin{aligned}
& C_{1}=(5 ; 2,2,2,2,1,2,2,1),(2,3) \\
& C_{2}=(5 ; 2,2,2,2,2,1,2,1),(2,3) \\
& C_{3}=(5 ; 2,2,2,1,2,2,2,1),(2,3)
\end{aligned}
$$




$$
\begin{aligned}
& C_{4}=(5 ; 2,2,2,2,2,2,1,1),(2,3) \\
& C_{5}=(4 ; 2,2,2,1,1,1,1,1),(2,2) \\
& C_{6}=(3 ; 1,1,2,1,1,1,1,0),(1,2) \\
& C_{7}=(3 ; 2,1,1,1,1,1,1,0),(1,2) \\
& C_{8}=(3 ; 1,2,1,1,1,1,1,0),(1,2)
\end{aligned}
$$

Now $\left(\left(a_{1}^{2}-a_{1}\right) / 2+\left(b_{1}^{2}-b_{1}\right) / 2\right)+\cdots+\left(\left(a_{n}^{2}-a_{n}\right) / 2+\left(b_{n}^{2}-b_{n}\right) / 2\right)$ here is 21 . But $(a-1)(a-2) / 2+(b-1)(b-2) / 2=20$ if we use $\left(a_{C}, b_{C}\right)=(6,6)$, so we see that $\left(a_{C}, b_{C}\right)=(5,7)$; this is also what we get if we use Macaulay 2 GS] to compute $\left(a_{C}, b_{C}\right)$, using randomly chosen points (so this is a check but not a proof that $(a, b)=(5,7)$ here $)$.

Also, it follows from $(*)$ and $(* *)$ that $\operatorname{dim} \operatorname{cok} \mu_{i C}=\operatorname{dim} \operatorname{cok} \mu_{C ; C}+\cdots+$ $\operatorname{dim} \operatorname{cok} \mu_{C ; i C}$ for all $i$ if $(* *)$ is an equality.

Thus (**) can, conjecturally, sometimes tell us both what the splitting type is and what the cokernel is. Moreover, $(* *)$ sometimes also applies to exceptional curves. In the example above, $E=C-E_{9}-E_{10}$ is exceptional and has the same splitting type as does $C$, so we get information about $E$ via $C$.

We have applied our script for computing splitting types to numerous examples. In these examples, $\left(a_{C}, b_{C}\right)$ always made $\left(a_{C}-1\right)\left(a_{C}-2\right) / 2+\left(b_{C}-1\right)\left(b_{C}-2\right) / 2$ as small as possible subject to $(* *)$. (Moreover, in those cases where $(* *)$ was not an equality, it was off by exactly 1 , and in those cases it always happened that $a_{i}=b_{i}$ for all $i$.) This and Lemma 2.1.2 lead us to make the following conjecture:

Conjecture A2.1.1. Let $C=w L$ for some $w \in W(X)$, let $d=C \cdot L$, let $m$ be the maximum of $C \cdot E_{1}, \ldots, C \cdot E_{n}$ and let $C_{1}=w E_{1}, \ldots, C_{n}=w E_{n}$. Then $\left(a_{C}, b_{C}\right)$ is the solution $(a, b)$ to $a \leq b, \min (m, d-m) \leq a \leq d-m$ and $d=a+b$ which minimizes $(a-1)(a-2) / 2+(b-1)(b-2) / 2$ subject to $(* *)$.

A2.2. Computational evidence for Conjecture 3.4. There are 2051 exceptional classes of the form $E=t L-m_{1} E_{1}-\cdots-m_{n} E_{n}$ with $1 \leq t \leq 20$ (taking $n$ to be as large as necessary) and $m_{1} \geq \cdots \geq m_{n} \geq 0$. We have applied our splitting script (using randomly chosen points $P_{i}$ and working in characteristic 31991) to determine the splitting types of all 2051. It turned out that Theorem 3.3 (b, c) implies for all but 25 of the 2051 cases that Conjecture 3.4 holds for the given E.

Theorem 3.3 $(\mathrm{b}, \mathrm{c})$ does not apply in the remaining 25 cases. Here we list these 25 cases, giving $a_{E}, b_{E}, E \cdot L$ and $E \cdot E_{i}$ for all $i$ such that $E \cdot E_{i}>0$ :

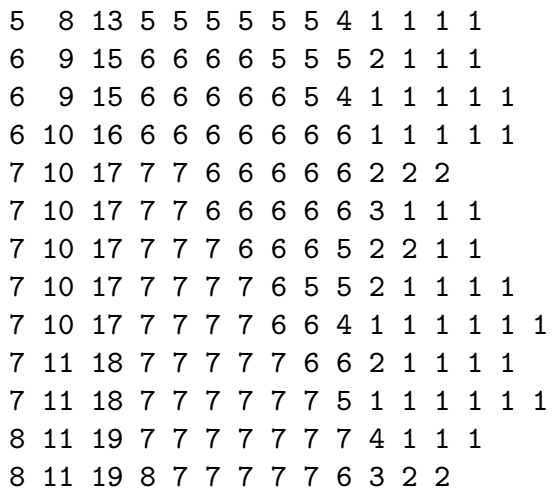

$\begin{array}{llllllllllllll}5 & 8 & 13 & 5 & 5 & 5 & 5 & 5 & 5 & 4 & 1 & 1 & 1 & 1\end{array}$

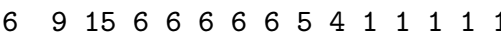

$\begin{array}{lllllllllllllll}6 & 10 & 16 & 6 & 6 & 6 & 6 & 6 & 6 & 6 & 1 & 1 & 1 & 1 & 1\end{array}$

22

$\begin{array}{llllllllllllll}7 & 10 & 17 & 7 & 7 & 7 & 6 & 6 & 6 & 5 & 2 & 2 & 1 & 1\end{array}$

$\begin{array}{lllllllllllllll}7 & 10 & 17 & 7 & 7 & 7 & 7 & 6 & 5 & 5 & 2 & 1 & 1 & 1 & 1\end{array}$

$\begin{array}{lllllllllllllllll}7 & 10 & 17 & 7 & 7 & 7 & 7 & 6 & 6 & 4 & 1 & 1 & 1 & 1 & 1 & 1\end{array}$

$\begin{array}{lllllllllllllllll}7 & 11 & 18 & 7 & 7 & 7 & 7 & 7 & 6 & 6 & 2 & 1 & 1 & 1 & 1\end{array}$

$\begin{array}{llllllllllllll}8 & 11 & 19 & 7 & 7 & 7 & 7 & 7 & 7 & 7 & 4 & 1 & 1 & 1\end{array}$

$\begin{array}{lllllllllllll}8 & 11 & 19 & 8 & 7 & 7 & 7 & 7 & 7 & 6 & 3 & 2 & 2\end{array}$

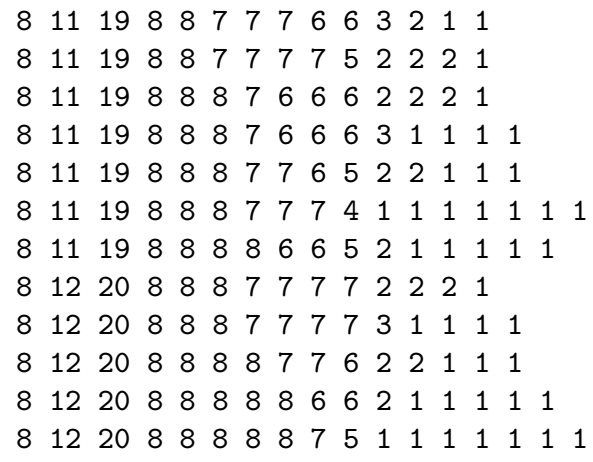


These cases can be checked by directly computing $\operatorname{dim} \operatorname{cok} \mu_{F}$ for $F=L+i E$. The critical value of $i$ is $i=b_{E}$ (if Conjecture 3.4 holds for $L+b_{E} E$, it follows from the sequences $(\ddagger)$ that it holds for $L+i E$ for all $0 \leq i \leq E \cdot L)$. In principle, one can compute dim $\operatorname{cok} \mu_{F}$ by computing a resolution of $I(Z)$ for $Z=m_{1} P_{1}+\cdots+m_{n} P_{n}$ in the usual way, using Gröbner bases. But for all but one of the 25 examples above we found this computation too large to successfully complete using this usual approach. By taking a different geometrically inspired approach we have in fact been able to check that Conjecture 3.4 holds in these 24 other cases. We now discuss this alternate approach for computing the dimension of the cokernel of $\mu_{L+m E}$ when $E$ is an exceptional curve and $0 \leq m \leq L \cdot E$. Since $\mu_{L+m E}$ and $\mu_{m E ; L+m E}$ have isomorphic cokernels, it is enough to compute the dimension of the cokernel of the latter, which it turns out one can do fairly efficiently, regarding the scheme structure of $m E$ as Proj of a certain graded ring. So first we determine this scheme structure.

Let $E \subset X$ be smooth and rational with $E^{2}=-1$. First, we would like to know $\operatorname{Pic}(m E)$, and to determine $h^{i}(m E, \mathcal{F})$ for both $i=0$ and $i=1$ for every line bundle $\mathcal{F}$ on $m E$.

Using exact sequences of the form $0 \rightarrow \mathcal{O}_{X}(d L+(j-m) E) \rightarrow \mathcal{O}_{X}(d L+j E) \rightarrow$ $\mathcal{O}_{m E}(d L+j E) \rightarrow 0$ and $0 \rightarrow \mathcal{O}_{X}((d-1) L+j E) \rightarrow \mathcal{O}_{X}(d L+j E) \rightarrow \mathcal{O}_{L}(d L+j E) \rightarrow$ 0 and the cohomology of divisors of the form $d L+j E$, which is known for all $d$ and $j$, we find that: $h^{0}\left(m E, \mathcal{O}_{m E}(d L+j E)\right)-h^{1}\left(m E, \mathcal{O}_{m E}(d L+j E)\right)=\left(\begin{array}{c}m+1 \\ 2\end{array}\right)+m t$ holds for all $t=E \cdot(d L+j E) ; h^{0}\left(m E, \mathcal{O}_{m E}(d L+j E)\right)=\left(\begin{array}{c}m+1 \\ 2\end{array}\right)+m t$ (and hence $\left.h^{1}\left(m E, \mathcal{O}_{m E}(d L+j E)\right)=0\right)$ and $h^{0}\left(m E, \mathcal{O}_{m E}(-d L-j E)\right)=\left(\begin{array}{c}m-t+1 \\ 2\end{array}\right)$ hold if $t \geq 0$; and it follows that $h^{1}\left(m E, \mathcal{O}_{m E}(-d L-j E)\right)=\left(\begin{array}{l}t \\ 2\end{array}\right)$ holds if $0 \leq t \leq m$, and $h^{1}\left(m E, \mathcal{O}_{m E}(-d L-j E)\right)=t m-\left(\begin{array}{c}m+1 \\ 2\end{array}\right)$ holds if $t \geq m$.

In particular, $h^{1}\left(m E, \mathcal{O}_{m E}\right)=0$, so it follows that the inclusion $E \subset m E$ induces an isomorphism $\operatorname{Pic}(m E) \rightarrow \operatorname{Pic}(E)=\mathbf{Z}$ (see $\underline{\text { A.Ar }}$, (1.3) and (1.4)). It now follows for any line bundle $F$ on $X$ that $h^{i}\left(m E, \mathcal{O}_{m E}(F)\right)$ depends only on $m, i$ and $E \cdot F$. In particular, $h^{i}\left(m E, \mathcal{O}_{m E}(F)\right)=h^{i}\left(m E, \mathcal{O}_{m E}(t)\right)$, where $t=E \cdot F$, so we define $\mathcal{O}_{m E}(1)$ to be $\mathcal{O}_{m E}(-E)$, and write $h^{i}(m E, t)$ for $h^{i}\left(m E, \mathcal{O}_{m E}(t)\right)$.

To get the scheme structure, we can pick any $E$ which is convenient, since $m E$ is isomorphic for all $E$ on $X$. Let $\pi: S \rightarrow T$ be the blowup of the point $P$ defined by $x=0=y$ in $T=\operatorname{Spec}(K[x, y])$, and take $E=\pi^{-1}(P)$. We can regard $S$ as $\operatorname{Proj}_{A}(A[u, v] /(x v-u y))$, where $A=K[x, y]$, and $E$ as $\operatorname{Proj}_{A}(A[u, v] /((x v-u y)+$ $(x, y)))$, which is just $E=\operatorname{Proj}_{K}(K[u, v])$. Similarly, $m E$ is the fiber $\pi^{-1}(m P)$, so $m E=\operatorname{Proj}_{A}\left(A[u, v] /\left((x v-u y)+(x, y)^{m}\right)\right)$. Note that the ring $B=A[u, v] /$ $\left((x v-u y)+(x, y)^{m}\right)$ is graded, with $x$ and $y$ of degree 0 and $u$ and $v$ of degree 1. The homogeneous component $B_{t}$ for $t \geq 0$ is just the span of the monomials $x^{i} y^{j} u^{r} v^{s}$ with nonnegative exponents where $r+s=t$, and $i+j<m$ (since we have modded out by $\left.(x, y)^{m}\right)$. In fact $B_{t}$ can be identified with $H^{0}(m E, t)$. However, $B$ has no components of negative degree, whereas $h^{0}(m E, t)>0$ for $t>-m$. But note that $x / u=y / v$ on the open set of $m E$ where neither $u$ nor $v$ is 0 . Thus taking $x / u$ where $u \neq 0$ and $y / v$ where $v \neq 0$ defines an element $\epsilon \in H^{0}(m E,-1)$. Let $C=K[u, v, \epsilon] /\left(\epsilon^{m}\right)$. This is graded if we take $\epsilon$ to have degree -1 . We have a graded injective ring homomorphism $B \rightarrow C$ given by sending $x \mapsto \epsilon u$ and $y \mapsto \epsilon v$, and now we can identify $C_{t}$ with $H^{0}(m E, t)$ for all $t$. In particular, the monomials of the form $\epsilon^{i} u^{r} v^{s}$ with nonnegative exponents satisfying $r+s=t+i$ and $i \leq m-1$ give a basis for $H^{0}(m E, t)$, and we can regard $m E$ as $\operatorname{Proj}_{K}(C)$. 
The map $\mu_{m E ; L+m E}$ factors via the restriction $H^{0}(X, L) \rightarrow H^{0}\left(m E, \mathcal{O}_{m E}(L)\right)$ through

$$
\mu: H^{0}\left(m E, \mathcal{O}_{m E}(L+m E)\right) \otimes H^{0}\left(m E, \mathcal{O}_{m E}(L)\right) \rightarrow H^{0}\left(m E, \mathcal{O}_{m E}(2 L+m E)\right) .
$$

Thus $\operatorname{Im} \mu_{m E ; L+m E}$ is the $K$-span of the product

$$
\left(H^{0}\left(m E, \mathcal{O}_{m E}(L+m E)\right)\right)\left(\operatorname{Im}\left(H^{0}(X, L) \rightarrow H^{0}\left(m E, \mathcal{O}_{m E}(L)\right)\right)\right)
$$

in $H^{0}\left(m E, \mathcal{O}_{m E}(2 L+m E)\right)$. It has the same dimension as does

$$
\left(H^{0}\left(m E_{1}, \mathcal{O}_{m E_{1}}\left(L^{\prime}+m E_{1}\right)\right)\right)\left(\operatorname{Im}\left(H^{0}\left(X, L^{\prime}\right) \rightarrow H^{0}\left(m E_{1}, \mathcal{O}_{m E_{1}}\left(L^{\prime}\right)\right)\right)\right)
$$

in $H^{0}\left(m E_{1}, \mathcal{O}_{m E_{1}}\left(2 L^{\prime}+m E_{1}\right)\right)$, where $w L=L^{\prime}$ for some appropriate Cremona transformation $w$ with $w E=E_{1}$.

Now choose coordinates on $\mathbf{P}^{2}$ such that $K\left[\mathbf{P}^{2}\right]=K[a, b, c]$, where $E_{1}$ is the blowup of the point $a=b=0$. Let $d=E_{1} \cdot L^{\prime}=E \cdot L$. Note that $L^{\prime}-$ $E_{1}$ is nef, so $0 \leq\left(L^{\prime}-E_{1}\right) \cdot L=L^{\prime} \cdot L-d$; thus $2 d-1 \leq d-1+L \cdot L^{\prime}$. Now, $H^{0}\left(m E_{1}, \mathcal{O}_{m E_{1}}\left(2 L^{\prime}+m E_{1}\right)\right)=H^{0}\left(m E_{1}, \mathcal{O}_{m E_{1}}(2 d-m)\right)$, so the monomials of the form $u^{i} v^{j} \epsilon^{k}$ with $2 d-m \leq i+j \leq 2 d-1$ and $0 \leq k=i+j-$ $(2 d-m)$ give a basis. Thus there is a surjective map of the homogeneous component $\left(\left((a, b, c)^{2 d-1}\right) \cap\left((a, b)^{2 d-m}\right)\right)_{d-1+L \cdot L^{\prime}}$ onto $H^{0}\left(m E_{1}, \mathcal{O}_{m E_{1}}(2 d-m)\right)$, defined by sending $a^{i} b^{j} c^{d-1-i-j+L \cdot L^{\prime}}$ to $u^{i} v^{j} \epsilon^{i+j-2 d+m}$, where $(a, b, c)$ denotes the ideal in $K[a, b, c]$ generated by $a, b$ and $c$. Moreover, the kernel of this surjective map is spanned by the monomials $a^{i} b^{j} c^{d-1-i-j+L \cdot L^{\prime}}$ with $i+j \geq 2 d$.

Note that the elements of $H^{0}\left(X, L^{\prime}\right)$, regarded as homogeneous polynomials in $K[a, b, c]$, are certain polynomials $f(a, b, c)$ of degree $L \cdot L^{\prime}$ such that the terms of $f(a, b, 1)$ of least degree have degree $d$. The image of $f(a, b, c)$ in $H^{0}\left(m E_{1}, \mathcal{O}_{m E_{1}}\left(L^{\prime}\right)\right)$ (i.e., the restriction of $f$ to $\left.m E_{1}\right)$ is just what you get if you formally simplify $\left(\epsilon^{d}\right)\left(f\left(u / \epsilon^{d}, v / \epsilon^{d}, 1\right)\right)$. Thus we have a surjection of $\left((a, b, c)^{d-1}\right) \cap\left((a, b)^{d-m}\right)$ onto $H^{0}\left(m E_{1}, \mathcal{O}_{m E_{1}}\left(L^{\prime}+m E_{1}\right)\right)$, defined by sending $a^{i} b^{j} c^{d-1-i-j}$ to $u^{i} v^{j} \epsilon^{i+j-d+m}$. This gives a surjective map of the homogeneous component

$$
\left(\left(\left((a, b, c)^{d-1}\right) \cap\left((a, b)^{d-m}\right)\right) H^{0}\left(X, L^{\prime}\right)+\left((a, b)^{2 d}\right)\right)_{d-1+L \cdot L^{\prime}}
$$

onto $\operatorname{Im} \mu_{m E_{1} ; L^{\prime}+m E_{1}}$ with kernel $\left((a, b)^{2 d}\right)_{d-1+L \cdot L^{\prime}}$. Thus $\operatorname{dim} \operatorname{Im} \mu_{m E_{1} ; L^{\prime}+m E_{1}}$ equals $\operatorname{dim}\left(\left(\left((a, b, c)^{d-1}\right) \cap\left((a, b)^{d-m}\right)\right) H^{0}\left(X, L^{\prime}\right)+\left((a, b)^{2 d}\right)\right)_{d-1+L \cdot L^{\prime}}$ $-\operatorname{dim}\left((a, b)^{2 d}\right)_{d-1+L \cdot L^{\prime}}$. The hardest part of this calculation is finding $H^{0}\left(X, L^{\prime}\right)$. It can be done either using a Gröbner basis calculation (but one much smaller than what is needed to calculate the dimension of the image of $\mu_{m E ; L+m E}$ directly) or by applying $w$ to a basis for $H^{0}(X, L)$.

\section{REFERENCES}

[AC] E. Arbarello and M. Cornalba, Footnotes to a paper of B. Segre, Math. Ann., 256 (1981), 341-362. MR626954 (83d:14016)

[As] M.-G. Ascenzi, The restricted tangent bundle of a rational curve in $\mathbf{P}^{2}$, Comm. Algebra 16 (1988), no. 11, 2193-2208. MR962311 (89i:14023)

[BI] E. Ballico and M. Idà, On the minimal free resolution for fat point schemes of multiplicity at most 3 in $\mathbf{P}^{2}$, J. Pure Appl. Algebra 212 (2008), 1756-1769.

[BZ] A. Buckley and M. Zompatori, Linear systems of plane curves with a composite number of base points of equal multiplicity, Trans. Amer. Math. Soc. 355 (2003), no. 2, 539-549. MR.1932712 (2003j:14039)

[Cas] G. Castelnuovo, Ricerche generali sopra i sistemi lineari di curve piane, Mem. Accad. Sci. Torino, II 42 (1891). 
[Cat] M. V. Catalisano, "Fat" points on a conic, Comm. Alg. 19(8) (1991), 2153-2168. MR.1123117 (93b:14016)

[CM1] C. Ciliberto and R. Miranda, Degenerations of planar linear systems, J. Reine Angew. Math. 501 (1998), 191-220. MR.1637857 (2000m:14005)

[CM2] C. Ciliberto and R. Miranda, Linear systems of plane curves with base points of equal multiplicity, Trans. Amer. Math. Soc. 352 (2000), no. 9, 4037-4050. MR1637062 (2000m:14006)

[CM3] C. Ciliberto and R. Miranda. Nagata's conjecture for a square or nearly-square number of points, Ric. Mat. 55 (2006), no. 1, 71-78. MR2248163 (2007d:14098)

[CCMO] C. Ciliberto, F. Cioffi, R. Miranda and F. Orecchia, Bivariate Hermite interpolation and linear systems of plane curves with base fat points, in: Computer mathematics, 87-102, Lecture Notes Ser. Comput., 10, World Sci. Publishing, River Edge, NJ, 2003. MR 2061827 (2005c:41002)

[dF] T. de Fernex, Negative curves on very general blow-ups of $\mathbf{P}^{2}$, Projective varieties with unexpected properties, 199-207, Walter de Gruyter GmbH \& Co. KG, Berlin, 2005. MR 2202253 (2006k:14018)

[E1] L. Evain, La fonction de Hilbert de la réunion de $4^{h}$ gros points génériques de $\mathbf{P}^{2}$ de même multiplicité, J. Algebraic Geom. 8 (1999), no. 4, 787-796. MR 1703614 (2000e:13023)

[E2] L. Evain, Computing limit linear series with infinitesimal methods, Ann. Inst. Fourier 57 (2007), 1947-1974. MR2377892

[F1] S. Fitchett, On Bounding the Number of Generators for Fat Point Ideals on the Projective Plane, J. Algebra, 236 (2001), 502-521. MR1813489(2002c:13028)

[F2] S. Fitchett, Corrigendum to: "On bounding the number of generators for fat point ideals on the projective plane” [J. Algebra 236 (2001), no. 2, 502-521], J. Algebra 276 (2004), no. 1, 417-419. MR2054405 (2005a:13029)

[F3] S. Fitchett, Maps of linear systems on blow-ups of the projective plane, J. Pure Appl. Algebra 156 (2001), 1-14. MR.1807013 (2002c:14013)

[FHH] S. Fitchett, B. Harbourne and S. Holay, Resolutions of Fat Point Ideals Involving Eight General Points of $\mathbf{P}^{2}$, J. Algebra 244 (2001), 684-705. MR1859044 (2002g:14089)

[GGR] A. V. Geramita, D. Gregory and L. Roberts, Monomial ideals and points in projective space, J. Pure Appl. Alg. 40 (1986), 33-62. MR825180 (87d:13023)

[G] A. Gimigliano, On linear systems of plane curves, Thesis, Queen's University, Kingston (1987).

[GHI1] A. Gimigliano, B. Harbourne and M. Idà, The role of the cotangent bundle in resolving ideals of fat points in the plane, in press, J. Pure Appl. Algebra (2008).

[GHI2] A. Gimigliano, B. Harbourne and M. Idà, Betti numbers for fat point ideals in the plane: A geometric approach, arXiv:0706.2588 [The source $\mathrm{T}_{\mathrm{E} X}$ file includes nonprinting explicit Macaulay 2 scripts for carrying out computations and for computing splitting types.]

[GI] A. Gimigliano and M. Idà, The ideal resolution for generic 3-fat points in $\mathbf{P}^{2}$, J. Pure Appl. Algebra 187 (2004), no. 1-3, 99-128. MR2027898(2005e:13017)

[GS] D. Grayson, and M. Stillman, Macaulay 2, a software system for research in algebraic geometry, Available at http://www.math.uiuc.edu/Macaulay2/.

[Ha1] B. Harbourne, An Algorithm for Fat Points on $\mathbf{P}^{2}$, Can. J. Math. 52 (2000), 123-140. MR.1745704 (2001g:13037)

[Ha2] B. Harbourne, The Ideal Generation Problem for Fat Points, J. Pure Appl. Alg. 145(2), 165-182 (2000). MR1733250 (2001c:13028)

[Ha3] B. Harbourne, Free Resolutions of Fat Point Ideals on $\mathbf{P}^{2}$, J. Pure Appl. Alg. 125, 213-234 (1998). MR1600024 (99d:13016)

[Ha4] B. Harbourne, The geometry of rational surfaces and Hilbert functions of points in the plane. Can. Math. Soc. Conf. Proc., vol. 6 (1986), 95-111. MR846019 (87k:14041)

[Ha5] B. Harbourne, Very ample divisors on rational surfaces, Math. Ann. 272, 139-153 (1985). MR794097 (86k:14026)

[HHF] B. Harbourne, S. Holay and S. Fitchett, Resolutions of ideals of quasiuniform fat point subschemes of $\mathbf{P}^{2}$, Trans. Amer. Math. Soc. 355 (2003), no. 2, 593-608. MR 1932715 (2004c:13019)

[HR] B. Harbourne and J. Roé. Linear systems with multiple base points in $\mathbf{P}^{2}$, Adv. Geom. 4 (2004), 41-59. MR2155364 (2006b:13038) 
[Hi1] A. Hirschowitz, Une conjecture pour la cohomologie des diviseurs sur les surfaces rationelles génériques, Journ. Reine Angew. Math. 397 (1989), 208-213. MR993223 (90g:14021)

[Hi2] A. Hirschowitz. La méthode d'Horace pour l'interpolation à plusieurs variables, Manus. Math. 50 (1985), 337-388. MR784148 (86j:14013)

[Ia] A. Iarrobino, Inverse system of a symbolic power III: Thin algebras and fat points, Compositio Math. 108 (1997), no. 3, 319-356. MR1473851 (98k:13017)

[I] M. Idà, The minimal free resolution for the first infinitesimal neighborhoods of n general points in the plane, J. Alg. 216 (1999), 741-753. MR.1692957 (2000d:13022)

[Mi] T. Mignon, Systèmes de courbes planes à singularités imposées: Le cas des multiplicités inférieures ou égales à quatre, J. Pure Appl. Algebra 151 (2000), no. 2, 173-195. MR:1775572 (2001g:14048)

[Mu1] D. Mumford, Varieties defined by quadratic equations, in: Questions on algebraic varieties, Corso C.I.M.E. 1969 Rome: Cremonese, 1970, 30-100. MR0282975 (44:209)

[Mu2] D. Mumford, Lectures on curves on an algebraic surface, Princeton 1966. MR0209285 $(35: 187)$

[N1] M. Nagata, On the 14-th problem of Hilbert, Amer. J. Math. 81 (1959), 766-772. MR 0105409 (21:4151)

[N2] M. Nagata, On rational surfaces, II, Mem. Coll. Sci. Univ. Kyoto, Ser. A Math. 33 (1960), 271-293. MR0126444(23:A3740)

[P] F. Palatini, Sulla rappresentazione delle forme ternerie mediante la somma di potenze di forme lineare, Atti Accad. Naz. Lincei Rend. (Serie V) vol. 12, n. 3 (1903).

[R] J. Roé, Limit linear systems and applications, preprint (math/0602213), 2006.

[S] B. Segre, Alcune questioni su insiemi finiti di punti in Geometria Algebrica, Atti del Convegno Internaz. di Geom. Alg., Torino (1961). MR0169119(29:6372)

[Y] S. Yang, Linear series in $\mathbf{P}^{2}$ with base points of bounded multiplicity, J. Algebraic Geom. 16 (2007), no. 1, 19-38. MR2257318 (2007i:14011)

Additional Citations for the Appendix:

[A.Ar] M. Artin, Some numerical criteria for contractability of curves on algebraic surfaces, Amer. J. Math. 84 (1962), 485-497. MR0146182 (26:3704)

[A.H1] B. Harbourne, Blowings-up of $\mathbf{P}^{2}$ and their blowings-down, Duke Math. J. 52, 129-148 (1985). MR791295 (86m:14026)

[A.H2] B. Harbourne, Complete linear systems on rational surfaces, Trans. Amer. Math. Soc. 289, 213-226 (1985). MR779061 (86h:14030)

Dipartimento di Matematica e CiRAM, Università di Bologna, 40126 Bologna, Italy

E-mail address: gimiglia@dm.unibo.it

Department of Mathematics, University of Nebraska, Lincoln, Nebraska 68588-0130

E-mail address: bharbour@math.unl.edu

Dipartimento di Matematica, Università di Bologna, 40126 Bologna, Italy

E-mail address: ida@dm.unibo.it 